\title{
CYCLIC COVERS OF NORMAL GRADED RINGS
}

\author{
Masataka Tomari and KeI-IChi Watanabe
}

\begin{abstract}
We give a description of a graded cyclic cover of a normal graded ring in terms of the Pinkham-Demazure description of normal graded rings $R=R(X, D)$. With the geometric description of $\mathrm{Cl}(R)$, it is shown that our cyclic cover $S$ possesses the Pinkham-Demazure description $S \cong R(Y, \tilde{D})$ [Theorem 1.3], by which we obtain a description of an index one cover [Corollary 1.7] of $R$. In $\S 2$, as an application of this description, we give criteria for the normal graded singularities to be Kawamata log terminal or to be $\log$ canonical. Further, in $\$ 3$ we study the relations between cyclic covers of the Kummer type and cyclic covers obtained by using Veronese subrings. Our results extend S. Mori's structure theorem regarding graded factorial domains.
\end{abstract}

\section{Introduction}

Cyclic covers of an algebraic variety have been found to be very important and have been used in many works. However, it is not easy to determine the properties of a cyclic cover explicitly in terms of given data. The aim of this paper is to give a description of a cyclic cover of a normal graded ring in terms of the Pinkham-Demazure description of normal graded rings, which uses a Weil divisor with rational coefficients. Here, our approach is to give a natural grading to our cyclic cover and find the Weil divisor with rational coefficients describing the new ring.

Let $R=\oplus_{k \geq 0} R_{k}$ be a normal graded ring that is a finitely generated algebra over a field $k$. As a geometric representation for such $R$, the following theorem, due to H. Pinkham in the two-dimensional case and M. Demazure in general, is fundamental.

THEOREM (Pinkham and Demazure [1, 11]). Let the situation be as described above. Let $T$ be a homogeneous element of degree one of the quotient field $Q(R)$ of $R$. Then $X$ is normal projective and there exists an ample $Q$-Cartier divisor $D$ on $X=\operatorname{Proj}(R)$ which satisfies the relations $R_{n}=H^{0}\left(X, O_{X}(n D)\right) T^{n}$ for $n \in \boldsymbol{Z}$. Further this $D$ is uniquely determined by the choice of $T$.

With $D$ and $X$ as above, we denote $R$ as $R=R(X, D)$. In [20, 21], one of the present authors studied the divisor class group of $R$, conditions for $R$ to be

Received October 26, 2000; revised May 31, 2001. 
Cohen-Macaulay or Gorenstein and conditions for rational singularity in terms of the description given by the above theorem. Our interest in this paper is in the cyclic cover $S$ of $R$ defined by a $Q$-Cartier homogeneous divisor $\mathscr{D}(E) \in$ $\operatorname{HDiv}(R) \otimes \boldsymbol{Q}$ of order $r$. Using the geometric description of $\mathrm{Cl}(R)$, it can be shown that our cyclic cover $S$ is a normal graded ring. The Pinkham and Demazure theorem implies that we can find a normal projective variety $Y$ and an ample $Q$-Cartier divisor $\tilde{D}$ on $Y$ which give the description $S \cong R(Y, \tilde{D})$. Theorem 1.3 expresses $Y$ and $S$ in terms of $X, D$, and $E \in \operatorname{Div}(X) \otimes Q$. This allows us to investigate $S$ more effectively.

In $\S 1$, we discuss relations involving canonical modules of $S$ and $R$ [Theorem 1.5], and class groups of $S$ and $R$ [Section 1.8]. In particular we obtain a description of an index one cover [Corollary 1.7.1], or canonical cover, of $R$. In [19], J. Wahl described index one covers of certain 2-dimensional singularities, considering the exceptional locus of resolution of the singularity. An advantage of our approach is that it allows us to obtain ring theoretic information that is more precise than that given by the resolution of singularity.

In $\S 2$ we give criteria for the normal graded singularities to be Kawamata $\log$ terminal or to be log canonical in terms of Pinkham-Demazure's construction using the calculation of $a^{\prime}$ introduced in 1.7. Since log terminal and $\log$ canonical singularities are determined by the properties of the index one cover, our theory plays an essential role.

In $\$ 3$ we study relations between the cyclic covers of the Kummer type, $S=R[X] /\left(X^{m}-v\right)$, and cyclic covers obtained from Veronese subrings. Our results, Theorems 3.3, 3.4 and 3.6, extend S. Mori's structure theorem regarding graded factorial domains.

The invariant $a(R)$ of a graded ring $R$ defined in [5] plays an important role in conditions for rational and related singularities. In this paper the integers $a^{\prime}$, which are related to $a(R)$ with regard to their roles in various contexts, also play an important role. Throughout this paper we assume that all rings and varieties are defined over a field. We also assume the normality of various rings and cyclic covers of them. For careful treatments of normality we refer to our previous paper [18].

Acknowledgment. For the preparation of this paper we carried out calculations for many examples with much helps from the software Ubasic 86 developed by Y. Kida, with which we could easily treat rational coefficient divisors. Our thanks are due to Prof. Y. Kida.

\section{\$1. Graded cyclic covers of normal graded rings in terms of the construction of Pinkham and Demazure}

1.1. Basic facts. To state our result we first review the basic background of the Demazure construction from $[20,21]$. Let $X$ be a normal irreducible projective scheme of $\operatorname{dim} \geq 1$ over a field $k$, and $k(X)$ the rational function 
field of $X$. We denote the groups of Weil divisors on $X$ by $\operatorname{Div}(X)$. For $E \in \operatorname{Div}(X) \otimes \boldsymbol{Q}$, we can attach the divisorial sheaf $O_{X}(E)$. Now, let $D \in \operatorname{Div}(X) \otimes \boldsymbol{Q}$ be a $\boldsymbol{Q}$-Cartier ample divisor described as in the introduction and represented as $D=\sum_{V \in \operatorname{Irr}^{1}(X)}\left(p_{V} / q_{V}\right) V$ (with $p_{V}, q_{V} \in \boldsymbol{Z},\left(p_{V}, q_{V}\right)=1, q_{V} \geq 1$ ). Here $\operatorname{Irr}^{1}(X)$ denotes the set of prime divisors of $X$. We are particularly interested in the denominators $q_{V}$ in this paper. We consider $\pi: C(X, D)=$ $\operatorname{Spec}_{X}\left(\bigoplus_{n \geq 0} O_{X}(n D) T^{n}\right) \rightarrow X$ and $\pi^{\prime}: U(X, D)=\operatorname{Spec}_{X}\left(\bigoplus_{n \in Z} O_{X}(n D) T^{n}\right) \rightarrow X$. Note that $C(X, D)$ and $U(X, D)$ are normal schemes. Then, we have the following commutative diagram.

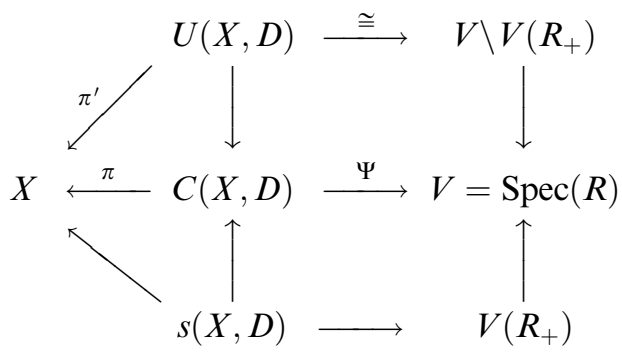

Here, $s(X, D)$ is the section of the $G_{m}$-fiber space $\pi: C(X, D) \rightarrow X$ and is defined by the ideal $\bigoplus_{n>1} O_{X}(n D) T^{n}$ of $O_{C(X, D)}$. In addition, $\Psi$ is the filtered blowingup of $\operatorname{Spec}(R)$ with respect to the filtration on $R$ induced by the grading of $R$.

For the divisor class group of these normal schemes, we have

$$
\mathrm{Cl}(R) \cong \mathrm{HDiv}(R) / \mathrm{HP}(R),
$$

where $\operatorname{HDiv}(R)$ (resp. $\operatorname{HP}(R))$ is the group of homogeneous (resp. homogeneous principal) divisors of $R$ (see [15]). We have the canonical isomorphism

$$
\operatorname{HDiv}(U) \cong \operatorname{HDiv}(R),
$$

where $\operatorname{HDiv}(U)$ is the group of the divisors on $U$ that are stable under the $\boldsymbol{G}_{m^{-}}$ action induced by the graded structure of $U=U(X, D)$. For a prime divisor $V$ of $X$, we set $F_{V}=\pi^{-1}(V)_{\text {red. }}$. Then we have $\pi^{*}(V)=q_{V} F_{V} \in \operatorname{HDiv}(U)$ ([1]). Now, we define the bijection

$$
\mathscr{D}: \operatorname{Div}(X) \otimes \boldsymbol{Q} \rightarrow \operatorname{HDiv}(U) \otimes \boldsymbol{Q} \cong \operatorname{HDiv}(R) \otimes \boldsymbol{Q}
$$

by $\mathscr{D}\left(\sum_{V \in \operatorname{Irr}^{1}(X)} r_{V} V\right)=\sum_{V \in \operatorname{Irr}^{1}(X)} q_{V} r_{V} F_{V}$. We define $\operatorname{Div}(X, D) \subset \operatorname{Div}(X) \otimes \boldsymbol{Q}$ by $\operatorname{Div}(X, D)=\mathscr{D}^{-1}(\operatorname{HDiv}(R))$. Considering the relation

$$
\operatorname{div}_{U}(T)=\pi^{\prime *}(D)=\sum_{V \in \operatorname{Irr}^{1}(X)} p_{V} F_{V} \in \operatorname{HDiv}(U)
$$

(see Proposition 2.9 of [1]) and the isomorphism $\operatorname{HP}(R) \cong P(X) \oplus \boldsymbol{Z} \operatorname{div}(T)$, we can see that $\mathscr{D}^{-1}(\operatorname{HP}(R))=P(X) \oplus Z D$, where $P(X)$ is the group of principal divisors on $X$. Hence we obtain the following:

$$
\mathrm{Cl}(R) \cong \operatorname{Div}(X, D) /(P(X) \oplus \boldsymbol{Z} D) .
$$


Further, we obtain the relations for the fractional graded $R$-ideals

$$
R(\mathscr{D}(E))=\bigoplus_{k \in \boldsymbol{Z}} \mathrm{H}^{0}\left(X, O_{X}(E+k D)\right) T^{k} \subset k(X)\left[T, T^{-1}\right]
$$

with $E \in \operatorname{Div}(X) \otimes Q$. In particular, $R\left(\mathscr{D}\left(-\left(1 / q_{V}\right) V\right)\right) \subset R$ is the prime ideal associated with $V \in \operatorname{Irr}^{1}(X)$.

1.2. Construction of a graded cyclic cover. Let $\mathscr{D}(E)$ be a $Q$-Cartier homogeneous $Q$-divisor of $R$ with $E \in \operatorname{Div}(X) \otimes Q$, and let $r>0$ be the minimal integer such that $r \mathscr{D}(E)=\mathscr{D}(r E) \in \operatorname{HP}(R)$. Then there exist $a^{\prime} \in \boldsymbol{Z}$ and $\varphi \in k(X)$ such that $r E-a^{\prime} D=\operatorname{div}_{X}(\varphi)$. We can easily see that $\varphi T^{a^{\prime}} R(r \mathscr{D}(E))=R$, and hence $\operatorname{div}_{R}\left(\varphi T^{a^{\prime}}\right)=r \mathscr{D}(E)$. Next, associated with the triple $\left(R, \mathscr{D}(E), \varphi T^{a^{\prime}}\right)$, we introduce the cyclic $r$-cover $S$ as follows (see [18]):

$$
S=S\left(R, \mathscr{D}(E), \varphi T^{a^{\prime}}\right)=\bigoplus_{i=0}^{r-1} R(i \mathscr{D}(E)) u^{i}, \quad \text { with } u^{r}=\varphi T^{a^{\prime}}
$$

We call such an $S$ a graded cyclic $r$-cover of $R$. If $\mathscr{D}(E)$ is an integral divisor of $R$ (that is, if $E \in \operatorname{Div}(X, D)$ ), we say that $S$ is an integral graded cyclic $r$-cover of $R$. Throughout this paper we assume that either (i) $\operatorname{char}(R)=0$ or (ii) $\operatorname{char}(R)=$ $p>0,(r, p)=1$ and every coefficient of $r \mathscr{D}(E)$ is an integer relatively prime with $p$. Then $S$ is a normal domain (Proposition 1.12 of [18]). If we set the degrees of $u$ and $T$ as $\operatorname{deg}(u)=a^{\prime} / s$ and $\operatorname{deg}(T)=r / s, S$ has a $Z$-graded structure, where $s=\left(r, a^{\prime}\right)$. Now, let $\alpha, \beta \in \boldsymbol{Z}$ satisfy $\alpha a^{\prime}+\beta r=s$, and define $\tilde{T}=T^{\beta} u^{a^{\prime}}$. Then $\tilde{T}$ is a homogeneous element of $Q(S)$ of degree 1. Now the Pinkham-Demazure description of $S$ is given by the following.

THEOREM 1.3. Let $S=S\left(R, \mathscr{D}(E), \varphi T^{a^{\prime}}\right)$ be the normal graded cyclic $r$-cover of $R=R(X, D)$ described above. Then, the Pinkham-Demazure description of $S$ with respect to $\tilde{T}=T^{\beta} u^{\alpha}$ with $\alpha a^{\prime}+\beta r=s\left(=\left(r, a^{\prime}\right)\right)$ is given by $S=R(Y, \tilde{D})$ as follows:

(1) $Y$ is the cyclic cover of $X$ given by $\rho: Y=\operatorname{Spec}_{X}\left(\oplus_{l=0}^{s-1} O_{X}(l((r / s) E-\right.$ $\left.\left.\left.\left(a^{\prime} / s\right) D\right)\right)\right) \rightarrow X$.

(2) $\tilde{D}=\rho^{*}(\alpha E+\beta D)$.

(3) We have the relations $\rho^{*}(E)-\left(a^{\prime} / s\right) \tilde{D} \in P(Y)$ and $\rho^{*}(D)-(r / s) \tilde{D} \in P(Y)$.

Proof. (1) Since $\sqrt{R_{+} S}=S_{+}$, it follows that $\operatorname{Proj}(S)$ is covered by the schemes of the form $\operatorname{Spec}\left(\left(S_{f_{d}}\right)_{0}\right)$, with $f_{d} \in R_{d}(d>0)$. Here we have the relation

$$
\widetilde{S_{f_{d}}}=\left.\left(\bigoplus_{i=0}^{r-1}\left(\bigoplus_{k \in Z} O_{X}(i E+k D) T^{k}\right) u^{i}\right)\right|_{\operatorname{Spec}\left(\left(R_{f_{d}}\right)_{0}\right)} \quad \text { on } \operatorname{Spec}\left(\left(R_{f_{d}}\right)_{0}\right) \subset X \text {. }
$$

Since $T^{k} u^{i}$ has degree 0 iff $k+i\left(a^{\prime} / r\right)=0$, there are exactly $s$ possibilities for such pairs $(k, i)$, given by $\left(-\left(l a^{\prime} / s\right),(l r / s)\right)$ for $l=0, \ldots, s-1$. Thus we have 


$$
Y=\operatorname{Proj}(S) \cong \operatorname{Spec}_{X}\left(\bigoplus_{l=0}^{s-1} O_{X}\left(l\left(\frac{r}{s} E-\frac{a^{\prime}}{s} D\right)\right) T^{-\left(l a^{\prime} / s\right)} u^{l r / s}\right) .
$$

(2) Considering the part of $\widetilde{S_{f_{d}}}$ of degree $n$, we have

$$
O_{Y}(n \tilde{D})\left(T^{\beta} u^{\alpha}\right)^{n} \cong \bigoplus_{l=0}^{s-1} O_{X}\left(l\left(\frac{r}{s} E-\frac{a^{\prime}}{s} D\right)+n(\alpha E+\beta D)\right) T^{-\left(l a^{\prime} / s\right)} u^{l r / s}\left(T^{\beta} u^{\alpha}\right)^{n}
$$

This implies the relation $\tilde{D}=\rho^{*}(\alpha E+\beta D)$.

(3) This follows from the relations $\rho^{*}(E)-\left(a^{\prime} / s\right) \rho^{*}(\alpha E+\beta D)=\beta \rho^{*}((r / s) E-$ $\left.\left(a^{\prime} / s\right) D\right)$ and $\rho^{*}(D)-(r / s) \rho^{*}(\alpha E+\beta D)=\alpha \rho^{*}\left((r / s) E-\left(a^{\prime} / s\right) D\right)$.

Q.E.D.

Remark 1.4. (1) In Theorem 1.3, if we choose one couple $(\alpha, \beta)$, another possible choice, $\left(\alpha^{\prime}, \beta^{\prime}\right)$, is $\left(\alpha^{\prime}, \beta^{\prime}\right)=\left(\alpha-r / s, \beta+a^{\prime} / r\right)$. Then $\alpha^{\prime} E+\beta^{\prime} D=(\alpha E+\beta D)-$ $\left((r / s) E-\left(a^{\prime} / s\right) D\right)$ on $X$. Thus certainly $\rho^{*}\left(\alpha^{\prime} E+\beta^{\prime} D\right)$ and $\rho^{*}(\alpha E+\beta D)$ are linearly equivalent on $Y$.

(2) Here, by the definition of the grading on $S, R$ is the $r$ th Veronese subring of $S$ if and only if $s=1$.

To facilitate discussion of the canonical module, we introduce the following notation: For any $\boldsymbol{Q}$-divisor $G=\sum\left(p_{W} / q_{W}\right) W$ (with $p_{W}, q_{W} \in \boldsymbol{Z},\left(p_{W}, q_{W}\right)=1$, $\left.q_{W} \geq 1\right)$, we define $G^{\prime}$ by $G^{\prime}=\sum\left(\left(q_{W}-1\right) / q_{W}\right) W$. As shown in [20], the canonical module $K_{R}$ of $R=R(X, D)$ is given by $K_{R}=R\left(\mathscr{D}\left(K_{X}+D^{\prime}\right)\right)$. Now, $K_{X}+D^{\prime}$ and $K_{Y}+(\tilde{D})^{\prime}$ are related by the following.

TheOREM 1.5. Let $S\left(R(X, D), \mathscr{D}(E), \varphi T^{a^{\prime}}\right)=R(Y, \tilde{D})$ be as in Theorem 1.3. Then we have

$$
\rho^{*}\left(K_{X}+D^{\prime}+\mathscr{D}^{-1}\left(\mathscr{D}(E)^{\prime}\right)\right)-\left(K_{Y}+(\tilde{D})^{\prime}\right) \in P(Y) .
$$

Proof. By Theorem 3.2 of [18], we have

$$
K_{S}=\bigoplus_{i=0}^{r-1} K_{R}\left(\mathscr{D}(E)^{\prime}+k \mathscr{D}(E)\right) u^{i}=\bigoplus_{i=0}^{r-1} R\left(\mathscr{D}\left(K_{X}+D^{\prime}\right)+\mathscr{D}(E)^{\prime}+k \mathscr{D}(E)\right) u^{i} .
$$

By arguments similar to those in the proof of Theorem 1.3, we can show the relation

$$
\bigoplus_{n \in Z} O_{Y}\left(\mathscr{D}_{S}^{-1}\left(K_{S}\right)+n \tilde{D}\right) \cong \bigoplus_{n \in Z} O_{Y}\left(\rho^{*}\left(K_{X}+D^{\prime}+\mathscr{D}^{-1}\left(\mathscr{D}(E)^{\prime}\right)\right)+n \tilde{D}\right) .
$$

Here the bijection $\mathscr{D}_{S}: \operatorname{Div}(Y) \otimes \boldsymbol{Q} \rightarrow \operatorname{HDiv}(S)$ is defined in the same way as $\mathscr{D}$ of $R$. Studying the ramifications of $\xi: \operatorname{Div}(R) \rightarrow \operatorname{HDiv}(S)$ (cf. [18; Lemma 1.10, (2.4)]), it can be shown that $(\xi \otimes \boldsymbol{Q})\left(\mathscr{D}(E)^{\prime}\right) \in \operatorname{HDiv}(S)$. Then, comparing the ramifications $X \rightarrow R \rightarrow S$ and $X \rightarrow Y \rightarrow S$, we can see that $\rho^{*}\left(\mathscr{D}^{-1}\left(\mathscr{D}(E)^{\prime}\right)\right) \in$ $\operatorname{Div}(Y, \tilde{D})$. We can also see $\rho^{*}\left(K_{X}+D^{\prime}\right) \in \operatorname{Div}(Y, \tilde{D})$, and hence $\rho^{*}\left(K_{X}+D^{\prime}+\right.$ $\left.\mathscr{D}^{-1}\left(\mathscr{D}(E)^{\prime}\right)\right) \in \operatorname{Div}(Y, \tilde{D})$. Thus the assertion follows from the bijectivity of $\mathscr{D}_{S}: \operatorname{Div}(Y, \tilde{D}) \rightarrow \operatorname{Div}(U(Y, \tilde{D}))$.

Q.E.D. 
Remark 1.6. (1) If we represent $E$ as $E=\sum_{V \in \operatorname{Irr}^{1}(X)}\left(p_{E, V} / q_{E, V}\right) V$, with $p_{E, V}, q_{E, V} \in \boldsymbol{Z},\left(p_{E, V}, q_{E, V}\right)=1$, and $q_{E, V} \geq 1$, then we can easily see that

$$
\mathscr{D}^{-1}\left(\mathscr{D}(E)^{\prime}\right)=\sum_{V \in \operatorname{Irr}^{1}(X)} \frac{\operatorname{LCM}\left(q_{V}, q_{E, V}\right) / q_{V}-1}{\operatorname{LCM}\left(q_{V}, q_{E, V}\right)} V,
$$

and hence

$$
D^{\prime}+\mathscr{D}^{-1}\left(\mathscr{D}(E)^{\prime}\right)=\sum_{V \in \operatorname{Irr}^{1}(X)} \frac{\operatorname{LCM}\left(q_{V}, q_{E, V}\right)-1}{\operatorname{LCM}\left(q_{V}, q_{E, V}\right)} V .
$$

In $\S 2$, we discuss the klt conditions of $K_{X}+D^{\prime}+\mathscr{D}^{-1}\left(\mathscr{D}(E)^{\prime}\right)$.

(2) Let $\tilde{V}$ be the prime divisor of $Y$ that lies over a prime divisor $V$ of $X$. Then, by Theorem 1.5, we can see that the denominator of the fractional part of $\tilde{D}$ is given by $\operatorname{LCM}\left(q_{V}, q_{E, V}\right) / e_{\tilde{V} / V}$, where $e_{\tilde{V} / V}$ is the ramification index of $\rho: Y \rightarrow X$ along $\tilde{V}$.

1.7. Index one cover. Now, suppose that $K_{R}+\mathscr{D}(E)$ is a $\boldsymbol{Q}$-Cartier divisor of index $r$. There are $a^{\prime} \in \boldsymbol{Z}$ and $\varphi \in k(X)$ such that $r\left(K_{X}+D^{\prime}+E\right)-a^{\prime} D=$ $\operatorname{div}_{X}(\varphi)$. In the case $\mathscr{D}(E)=0$, the integral graded $r$-cyclic cover $S=$ $S\left(R, \mathscr{D}\left(K_{X}+D^{\prime}\right), \varphi T^{a^{\prime}}\right)$ is called an index one cover of $R$ which is very important in the theory of singularities. There are already several works on singularities appearing in the index one covers of singularities $[12,13,19]$.

COROLlary 1.7.1. Let $S=S\left(R, K_{R}+\mathscr{D}(E), \varphi T^{a^{\prime}}\right)$ be the normal graded cyclic r-cover of $R=R(X, D)$ as described above. Then the Pinkham-Demazure construction $S$ with respect to $\tilde{T}=T^{\beta} u^{\alpha}$ with $\alpha a^{\prime}+\beta r=s\left(=\left(r, a^{\prime}\right)\right)$ is given by $S=R(Y, \tilde{D})$ as follows:

(1) $Y$ is the cyclic cover of $X$ given by

$$
\rho: Y=\operatorname{Spec}_{X}\left(\bigoplus_{l=0}^{s-1} O_{X}\left(l\left(\frac{r}{s}\left(K_{X}+D^{\prime}+E\right)-\frac{a^{\prime}}{s} D\right)\right)\right) \rightarrow X .
$$

(2) $\tilde{D}=\rho^{*}\left\{\alpha\left(K_{X}+D^{\prime}+E\right)+\beta D\right\}$.

(3) If $\tilde{E}=\rho^{*}\left(E-\mathscr{D}^{-1}\left(\mathscr{D}(E)^{\prime}\right)\right)$, then $\tilde{E} \in \operatorname{Div}(Y, \tilde{D})$, and we obtain the relation $K_{S}\left(\mathscr{D}_{S}(\tilde{E})\right)=S\left(a^{\prime} / s\right)$.

Proof of (3). We have $\rho^{*}\left(K_{X}+D^{\prime}+E\right)-\left(a^{\prime} / s\right) \tilde{D} \in P(Y)$ by the assertion (3) of Theorem 1.3. Then, since $\mathscr{D}\left(K_{X}+D^{\prime}+E\right)^{\prime}=\mathscr{D}(E)^{\prime}$, we have $\rho^{*}\left(K_{X}+D^{\prime}+\mathscr{D}^{-1}\left(\mathscr{D}(E)^{\prime}\right)\right)-\left(K_{Y}+\tilde{D}^{\prime}\right) \in P(Y)$ by Theorem 1.5. Hence $K_{Y}+$ $\tilde{D}^{\prime}+\rho^{*}\left(E-\mathscr{D}^{-1}\left(\mathscr{D}(E)^{\prime}\right)\right)-\left(a^{\prime} / s\right) \tilde{D} \in P(Y)$.

Q.E.D.

1.8. Class groups. Let $S=S\left(R, \mathscr{D}(E), \varphi T^{a^{\prime}}\right)$ as in 1.2. To investigate the relations between $\mathrm{Cl}(R)$ and $\mathrm{Cl}(S)$, we need the homogeneous class group $\operatorname{HCl}(S)$ with respect to the $\boldsymbol{Z}_{r}$-grading of $S$ from $\$ 2$ of [18]. This is given by 


$$
\operatorname{HCl}(S)=\left\{\operatorname{cl}\left(\mu^{*}(F)\right) \in \operatorname{Cl}(S) \mid F \in \operatorname{Div}(R) \otimes Q, \mu^{*}(F) \in \operatorname{Div}(S)\right\},
$$

where $\mu^{*}: \operatorname{Div}(R) \otimes \boldsymbol{Q} \rightarrow \operatorname{Div}(S) \otimes \boldsymbol{Q}$ is the natural map. Then we have the exact sequence (see $[18: 2.6])$

$$
0 \rightarrow \boldsymbol{Z} \operatorname{cl}(L \mathscr{D}(E)) \rightarrow \mathrm{Cl}(R) \rightarrow \mathrm{HCl}(S) \rightarrow \operatorname{Coker} \beta \rightarrow 0,
$$

where $L$ and $\beta$ are defined as $L=\min \{l \in \boldsymbol{Z} \mid l>0, l \mathscr{D}(E) \in \operatorname{Div}(R)\}$ and $\beta$ : $\boldsymbol{Z} \rightarrow \oplus \boldsymbol{Z} / Q_{\mathscr{V}} \boldsymbol{Z}$ with $\beta(1)=\left(P_{\mathscr{V}}\right)$, where we represent $\mathscr{D}(E)$ as $\mathscr{D}(E)=$ $\sum\left(P_{\mathscr{r}} / Q_{\mathscr{r}}\right) \mathscr{V} \in \operatorname{Div}(R) \otimes \boldsymbol{Q}$ with $\quad P_{\mathscr{r}}, Q_{\mathscr{r}} \in \boldsymbol{Z}, \quad\left(P_{\mathscr{r}}, Q_{\mathscr{V}}\right)=1, \quad$ and $\quad Q_{\mathscr{r}} \geq 1$. Here $\mathscr{V}$ represents prime divisors of $R$, and the sum is over all such prime divisors. The following result plays an important role in the arguments given in $\S 3$.

Proposition 1.9. Let $S=S\left(R, \mathscr{D}(E), \varphi T^{a^{\prime}}\right) \cong R(Y, \tilde{D})$ be as in Theorem 1.3. Then, if $\left(r, a^{\prime}\right)=1$, we have $\mathrm{HCl}(S)=\mathrm{Cl}(S)$.

Proof. The relation $Y=X$ follows from Theorem 1.3. Also, we have the relations $\operatorname{Div}(X) \otimes \boldsymbol{Q} \supset \operatorname{Div}(X, \tilde{D}) \supset \operatorname{Div}(X, D)$. Hence $\mu^{*}(\operatorname{HDiv}(R) \otimes \boldsymbol{Q}) \supset$ $\operatorname{HDiv}(S)$. The assertion follows from the isomorphism $\mathrm{Cl}(S) \cong \operatorname{HDiv}(S) /$ $\operatorname{HP}(S)$.

Example 1.10. Let $S=\boldsymbol{R}[x, y, z] / x^{2}+y^{2}+z^{2}$. We know that $S$ is a UFD (see p. 34 of [15]) and it can be written $S=S\left(\boldsymbol{R}[x, y],(1 / 2) \operatorname{div}\left(x^{2}+y^{2}\right),-\left(x^{2}+y^{2}\right)\right)$. We have the relation $0=\mathrm{Cl}(\boldsymbol{R}[x, y])=\mathrm{HCl}(S)=\mathrm{Cl}(S)$. However, $r=a^{\prime}=s=2$. Hence the converse of Proposition 1.9 does not hold in general.

\section{§2. A characterization of the klt-conditions of normal graded rings}

2.1. The purpose of this section is to give criterion for the normal graded singularities to be log terminal or log canonical in terms of the PinkhamDemazure construction. The rationality is characterized in $[3,20,21]$, under the assumption that $\operatorname{Spec}(R)-V\left(R_{+}\right)$has only rational singularities. Here we discuss the rationality of $\operatorname{Spec}(R)-V\left(R_{+}\right)$in terms of the Pinkham-Demazure construction. Theorems 2.6 and 2.9 in this paper are natural continuation of studies in [20], [21] and the main result in $\S 4$ of [18]. In this section, the formula for Goto-Watanabe's $a(R)$ invariant related to the index one cover given in 1.7 is the key to reduce the situation considered here to index 1 . Now, we begin by considering finite cyclic covers. Throughout this section, all local rings are defined over an algebraically closed field of characteristic zero. Let us recall the definitions of $\log$ terminal singularity and $\log$ canonical singularity, which we need. We refer to the articles [8], [12] and [9] for more general facts about log terminal singularity and $\log$ canonical singularity. 
2.2. Definitions of klt and $\log$ canonical. Let $V=\operatorname{Spec}(R)$ be a normal local singularity of dimension $d$ with an effective rational divisor $\Delta \in \operatorname{Div}(R) \otimes \boldsymbol{Q}$. We wish to consider the situation in which the following holds.

(2.2.1) There is an integer $r$ such that $r\left(K_{V}+\Delta\right)$ is an integral Cartier divisor on $V$.

With this condition, let $\phi: \tilde{V} \rightarrow V$ be a resolution of singularities with normal crossing exceptional divisor $E=\phi^{-1}(\operatorname{Sing}(V))$ such that the total transform of $\Delta$ is a normal crossing divisor. Also, let $\tilde{\Delta}$ be the proper transform of $D$ by $\phi$. Define the divisor $F$ on $\tilde{V}$ by

$$
r\left(K_{\tilde{V}}+\tilde{\Delta}+E\right)=\phi^{*}\left(r\left(K_{V}+\Delta\right)\right)+F .
$$

(klt) $V$ is Kawamata log terminal (klt for short) with respect to $K_{V}+\Delta$ if $[\Delta] \leq 0$ (that is, all coefficients are less than 1), if the condition (2.2.1) is satisfied and if

(2.2.2) there is a resolution $\phi$ such that $F$ is an effective divisor whose support coincides with $E$.

(lc) $V$ is $\log$ canonical with respect to $K_{V}+\Delta$, if the condition (2.2.1) is satisfied and if

(2.2.3) there is a resolution $\phi$ such that $F$ is an effective divisor.

If (2.2.1) is satisfied, the smallest such positive number $r$ is called the $\left(K_{V}+\Delta\right)$-index .

To study the log terminal property and the log canonical property of singularities of $\boldsymbol{G}_{m}$-fiber spaces, we consider a particular finite cover that we now define. Let $R$ be a normal domain of dimension $d$ and define $S=S(R, D, f)$ as in [18], where $D$ is a fractional divisor of $R$, and $f \in K=Q(R)$, with $\operatorname{div}_{R}(f)=r \cdot D$. Moreover, we assume that

$$
r=\min \{i \in \boldsymbol{Z} \mid i>0 \text { and } i \cdot D \text { is a principal divisor }\} .
$$

As demonstrated in our previous paper [18], the cyclic cover $S$ is also a normal $d$-dimensional domain. Here we represent $D$ as $D=\sum_{V \in \operatorname{Irr}^{1}(R)}\left(p_{V} / q_{V}\right) V$, with $p_{V}, q_{V} \in \boldsymbol{Z}, q_{V} \geq 1$ and $\left(p_{V}, q_{V}\right)=1$. For $\rho: \operatorname{Spec}(S) \rightarrow \operatorname{Spec}(R)$ we have the following.

Lemma 2.3. Let $\Delta \in \operatorname{Div}(R) \otimes \boldsymbol{Q}$. (1) Then $\rho^{*}\left(K_{R}+\Delta\right)$ is a $\boldsymbol{Q}$-Cartier divisor on $S$ if and only if $K_{R}+\Delta$ is a $Q$-Cartier divisor on $R$. (2) $S$ is klt (resp. log canonical) with respect to $\rho^{*}\left(K_{R}+\Delta\right)$ if and only if $R$ is klt (resp. log canonical) with respect to $K_{R}+\Delta$.

Proof. (1) By $\S 2$ of [18], we have the exact sequence

$$
0 \rightarrow \boldsymbol{Z} \mathrm{cl}(L D) \rightarrow \mathrm{Cl}(R) \stackrel{\rho^{*}}{\rightarrow} \mathrm{Cl}(S),
$$

where $L$ is defined as $L=\min \{\ell \in \boldsymbol{Z} \mid \ell D \in \operatorname{Div}(R)\}$ and $\rho^{*}(b)=\bigoplus_{i=0}^{r-1} R(b+i D) u^{i}$ $\in \operatorname{Div}(S)$ for $b \in \operatorname{Div}(R)$. Let $m$ be a positive integer such that $m\left(K_{R}+\Delta\right) \in$ $\operatorname{Div}(R)$. From the above exact sequence, we can see that $\rho^{*}\left(m\left(K_{R}+\Delta\right)\right)$ is a 
$Q$-Cartier divisor of $S$ if and only if there is an integer $t^{\prime}>0$ such that $\operatorname{cl}\left(t^{\prime} m\left(K_{R}+\Delta\right)\right) \in \boldsymbol{Z} \operatorname{cl}(L D)$. Since $D$ is $\boldsymbol{Q}$-Cartier, we obtain the assertion.

For (2), the proof can now be carried out in a standard manner using Iitaka's log ramification formula [6, Theorem 11.5]. We omit the proof of this lemma (see $[8,9])$.

The rationality of $U(X, D)$ is studied already in our previous works; Lemma (3.1) and Example (3.5) of [20], $\$ 3$ and $\S 4$ of [22], §5 of [17], and Proposition (1.5) of [16]. The following result unifies various statements in [20, 21]. Our arguments follow almost the same line of reasoning as the proof of Theorem 3.12 of [18]. Before discussing the klt conditions, we make the following remark.

Lemma 2.4. Let $\mathscr{D}(E)$ be a homogeneous $Q$-divisor of $R(X, D)$ with $E \in \operatorname{Div}(X)$. Then $[\mathscr{D}(E)] \leq 0$ if and only if $\left[D^{\prime}+E\right] \leq 0$.

Proof. For any number $x$, we see that $q x<1$ if and only if $x+$ $(q-1) / q<1$.

Q.E.D.

Proposition 2.5. Let $R(X, D)$ be a normal graded ring over a field $k$ with $\operatorname{char}(k)=0$, and let $\mathscr{D}(E)$ be a homogeneous $\boldsymbol{Q}$-divisor with $E \in \operatorname{Div}(X) \otimes \boldsymbol{Q}$. Then $U(X, D) \cong \operatorname{Spec}(R(X, D))-V\left(R_{+}\right)$is klt (resp. log canonical) with respect to $K_{R}+\mathscr{D}(E)$ if and only if $(X, x)$ is klt (resp. log canonical) with respect to $K_{X, x}+D_{x}^{\prime}+E_{x}$ at all points $x \in X$.

Proof. Let $x \in X$ be a closed point of $X$. Then the fiber $U(X, D)_{x}$ of $U(X, D)$ over $\operatorname{Spec}\left(O_{X, x}\right)$ can be written $U(X, D)_{x}=\operatorname{Spec}_{O_{X, X}}(\mathscr{B})$, where

$$
\mathscr{B}=\bigoplus_{k \in \boldsymbol{Z}} O_{X, x}\left(k D_{x}\right) T^{k} \subset k(X)\left[T, T^{-1}\right]
$$

(cf. [22]). We choose $f_{x}$ and $r_{x}$ such that $\operatorname{div}_{O_{X, x}}\left(f_{x}\right)=r_{x} D_{x}$ in $\operatorname{Div}\left(O_{X, x}\right)$ and $r_{x}$ is the minimum at $O_{X, x}$. Then $f_{x}^{-1} T^{r_{x}} \in O_{X, x}\left(r_{x} D_{x}\right) T^{r_{x}}$ is a unit of $\mathscr{B}$. We obtain

$$
\mathscr{B} /\left(f_{x}^{-1} T^{r_{x}}-1\right) \mathscr{B} \cong S\left(O_{X, x}, D_{x}, f_{x}\right)=S \cong \bigoplus_{l=0}^{r_{x}-1} O_{X, x}\left(l D_{x}\right) T^{l} .
$$

Following Flenner [3], we define $\alpha: \mathscr{B} \rightarrow S\left[U, U^{-1}\right]=S \otimes_{k} k\left[U, U^{-1}\right]$ with $\alpha(g)=$ $\left\{g \bmod \left(f^{-1} T^{r_{x}}-1\right) \mathscr{B}\right\} \cdot U^{m}$ for $g \in O_{X, x}\left(m D_{x}\right) T^{m}$. Here, we have the relation

$$
\mathscr{B}\left(K_{\mathscr{B}}+\mathscr{D}(E)\right) \cdot S\left[U, U^{-1}\right]=S\left(\rho^{*}\left(K_{R}+D^{\prime}+E\right)\right) \otimes_{k} k\left[U, U^{-1}\right] .
$$

Since the characteristic of the base field is zero, $\alpha$ is étale $([3, \S 2])$. Hence $S$ is klt (resp. $\log$ canonical) with respect to $\rho^{*}\left(K_{R}+D^{\prime}+E\right)$ if and only if $U\left(O_{X, x}, D_{x}\right)$ is klt (resp. $\log$ canonical) with respect to $K_{\mathscr{B}}+\mathscr{D}(E)$. Hence the assertion follows from Lemma 2.3.

Q.E.D.

We can now show the main result of this section. 
THEOREM 2.6. Let $R=R(X, D)$ be a normal graded ring over a field $k$ with $\operatorname{char}(k)=0$, and let $\mathscr{D}(E)$ be a homogeneous $\boldsymbol{Q}$-divisor with $E \in \operatorname{Div}(X) \otimes \boldsymbol{Q}$ such that $[\mathscr{D}(E)] \leq 0$ (cf. Lemma 2.4). Also let $r \geq 1$ be an integer. Then $R(X, D)$ is klt with respect to $K_{R}+\mathscr{D}(E)$ of index $r$ if and only if the following two conditions hold.

(1) There exists $a^{\prime} \in \boldsymbol{Z}$ satisfying $a^{\prime} \leq-1$ such that $r\left(K_{X}+D^{\prime}+E\right)-a^{\prime} D$ is an integral principal divisor on $X$. Further $r \in N$ is the minimal integer such that this holds.

(2) At each point $x$ of $X,(X, x)$ is klt with respect to $K_{X, x}+D_{x}^{\prime}+E_{x}$.

Proof. The proof is given in two steps. First, we show the assertions under the assumption that $K_{R}+\mathscr{D}(E)$ is a Cartier divisor on $R$. In the second step, we demonstrate that the result of Step 1 proves the theorem by using the log index one cover given in Corollary 1.7.1.

SteP 1. Assume that $K_{R}+\mathscr{D}(E)$ is a Cartier divisor of $R$. Then, we may assume that $U(X, D)$ is klt with respect to $K_{R}+\mathscr{D}(E)$ by Proposition 2.5. As shown in $\S 11$ of $[9], U(X, D)$ has only rational singularities. From this point, the proof can be carried out in almost the same way as in $\S 3$ of [21].

By assumption, there are $a \in Z$ and $\varphi \in k(X)$ such that $K_{X}+D^{\prime}+E-a D=$ $\operatorname{div}_{X}(\varphi)$. Here $-\mathscr{D}(E)$ is an effective integral divisor. Hence $K_{S} \supset K_{S}(\mathscr{D}(E))=$ $\left(1 /\left(\varphi T^{a}\right)\right) S$. We denote the rational form $1 /\left(\varphi T^{a}\right)$ by $\omega_{0}$. Let $\Psi: C(X, D) \rightarrow$ $\operatorname{Spec}(R)$ be the graded blowing-up as in 1.1. Also, let $\mathscr{D}(E)_{1} \subset C(X, D)$ be the proper transform of $\mathscr{D}(E)$. Then, by Proposition (1.6) of [21], we obtain the following relations:

$$
\omega_{C}\left(\mathscr{D}(E)_{1}\right)=\bigoplus_{k \geq 1} O_{X}\left(K_{X}+D^{\prime}+E+k D\right) T^{k}=\Psi^{*}\left(\omega_{0}\right) O_{C}(-(a+1) X) .
$$

If $R$ is klt with respect to $K_{R}+\mathscr{D}(E)$, then $-a>0$ by definition. Hence the condition (1) follows. Conversely, assume that $a<0$. We have $\omega_{C}=\Psi^{*}\left(\omega_{0}\right)$. $O_{C}(-\mathscr{D}(E)-(a+1) X) \supset \Psi^{*}\left(\omega_{0}\right) O_{C}$. Hence $\Psi^{*}\left(\omega_{0}\right)$ is regular on $C$. Then, since $C$ has only rational singularities, $(\Psi \tau)^{*}\left(\omega_{0}\right)$ is regular for any resolution $\tau: \tilde{C} \rightarrow C$. We can now easily check the klt condition (2.2.2). This completes Step 1 of the proof.

SteP 2. Suppose $K_{R}+\mathscr{D}(E)$ is a $Q$-Cartier divisor of index $r$. Let $S=$ $S\left(R, K_{R}+D^{\prime}+E, \phi T^{a^{\prime}}\right)=R(Y, \tilde{D})$ be the associated graded cyclic $r$-cover as described in 1.7. Let $\eta: \operatorname{Spec}(S) \rightarrow \operatorname{Spec}(R)$ and $\rho: Y \rightarrow X$ be the associated cyclic covers.

By Corollary 1.7.1, $\eta^{*}\left(K_{R}+\mathscr{D}(E)\right)=K_{X}+\mathscr{D}_{S}(\tilde{E}) \quad$ with $\quad \tilde{E}=\rho^{*}(E-$ $\mathscr{D}^{-1}\left(\mathscr{D}(E)^{\prime}\right) \in \operatorname{Div}(Y, \tilde{D})$. The condition $\mathscr{D}_{S}(\tilde{E}) \leq 0$ holds, since we have assumed $[\mathscr{D}(E)] \leq 0$ (cf. Lemma 2.4). Hence $R$ is klt with respect to $K_{R}+\mathscr{D}(E)$ if and only if $S$ is klt with respect to $K_{S}+\mathscr{D}_{S}(\tilde{E})$, by Lemma 2.3 .

With the conditions for $S$ corresponding to (1) and (2) in the statement of the theorem, we have the relations 


$$
K_{Y}+\tilde{D}^{\prime}+\tilde{E}-\frac{a^{\prime}}{s} \tilde{D}=K_{Y}+\tilde{D}^{\prime}+\tilde{E}-\rho^{*}\left(K_{X}+D^{\prime}+E\right) \in P(Y) .
$$

Obviously $a^{\prime}<0$ if and only if $a^{\prime} / s<0$. Further, $X$ is klt with respect to $K_{X}+D^{\prime}+E$ if and only if $Y$ is klt with respect to $K_{Y}+\tilde{D}^{\prime}+\tilde{E}$, by Lemma 2.3 . Hence (1) and (2) hold for $R$ if and only if (1) and (2) hold for $S$. This completes the proof of Theorem 2.6.

Next we consider the necessary condition for $R$ to have a $\log$ canonical singularity.

Proposition 2.7. Let $R=R(X, D)$ be a normal graded ring over a field $k$ with $\operatorname{char}(k)=0$, and let $\mathscr{D}(E)$ be a homogeneous $\boldsymbol{Q}$-divisor with $E \in \operatorname{Div}(X) \otimes \boldsymbol{Q}$. If $R$ is log canonical with respect to $K_{R}+\mathscr{D}(E)$ of index $r$, represented by the Pinkham-Demazure construction, then the following two conditions hold.

(1) There exists $a^{\prime} \in \boldsymbol{Z}$ with $a^{\prime} \leq 0$ such that $r\left(K_{X}+D^{\prime}+E\right)-a^{\prime} D$ is an integral principal divisor on $X$, and $r \in \boldsymbol{N}$ is the minimal integer such that this holds.

(2) At each point $x$ of $X,(X, x)$ has a log canonical singularity with respect to $K_{X, x}+D_{x}^{\prime}+E_{x}$.

Proof. First, (2) follows from Proposition 2.5. Now assume $K_{R}+\mathscr{D}(E)$ is a $Q$-Cartier divisor of index $r$. Let $S=S\left(R, K_{R}+D^{\prime}+E, \phi T^{a^{\prime}}\right)=R(Y, \tilde{D})$ be the associated graded cyclic $r$-cover as described in 1.7. Let $\eta: \operatorname{Spec}(S) \rightarrow$ $\operatorname{Spec}(R)$ and $\rho: Y \rightarrow X$ be the associated cyclic covers.

By Corollary 1.7.1, $\eta^{*}\left(K_{R}+\mathscr{D}(E)\right)=K_{X}+\mathscr{D}_{S}(\tilde{E}), \quad$ with $\quad \tilde{E}=\rho^{*}(E-$ $\mathscr{D}^{-1}\left(\mathscr{D}(E)^{\prime}\right) \in \operatorname{Div}(Y, \tilde{D})$. Since $R$ is $\log$ canonical with respect to $K_{R}+\mathscr{D}(E)$, $S$ is $\log$ canonical with respect to $K_{S}+\mathscr{D}_{S}(\tilde{E})$, by Lemma 2.3. We have the relation $K_{Y}+\tilde{D}^{\prime}+\tilde{E}-\left(a^{\prime} / s\right) \tilde{D} \in P(Y)$. Let $\Psi: C(Y, \tilde{D}) \rightarrow \operatorname{Spec}(S)$ be the graded blowing-up as in 1.1. Let $\mathscr{D}_{S}(\tilde{E})_{1} \subset C(Y, \tilde{D})$ be the proper transform of $\mathscr{D}_{S}(\tilde{E})$. Then, as in Step 1 of the proof of Theorem 2.6, we obtain the relation $\omega_{C}\left(\mathscr{D}_{S}(\tilde{E})_{1}\right) \cong O_{C}\left(-\left(a^{\prime} / s+1\right) Y\right)$. Thus $a^{\prime} / s \leq 0$ follows from the definition of the log canonical condition of $K_{S}+\mathscr{D}_{S}(\tilde{E})$.

This completes the proof.

Although we have not been able to obtain a necessary and sufficient condition for $R(X, D)$ to be a $\log$ canonical singularity as in 2.6 , we do have a sufficient condition.

THEOREM 2.8. Let $R=R(X, D)$ be a normal graded ring over a field $k$ with $\operatorname{char}(k)=0$, and let $\mathscr{D}(E)$ be a homogeneous $\boldsymbol{Q}$-divisor with $E \in \operatorname{Div}(X) \otimes \boldsymbol{Q}$ such that $\mathscr{D}(E)=\mathscr{D}(E)^{\prime}$. Let $r>0$ be an integer. Suppose the following conditions hold.

(1) There exists $a^{\prime} \in Z$ with $a^{\prime} \leq 0$ such that $r\left(K_{X}+D^{\prime}+E\right)-a^{\prime} D$ is an integral principal divisor on $X$. Further $r \in N$ is the minimal integer such this holds.

(2) At each point $x$ of $X,(X, x)$ is klt with respect to $K_{X, x}+D_{x}^{\prime}+E_{x}$.

Then $R(X, D)$ is a log canonical singularity of index $r$ with respect to $K_{R}+\mathscr{D}(E)$. 
Proof (cf. [7, 16]). If $a^{\prime}<0$, then, as we have already seen, $R$ is a klt singularity of index $r$ with respect to $K_{R}+\mathscr{D}(E)$ by Theorem 2.6. Hence we assume $a^{\prime}=0$ in the following. As for the proof of Theorem 2.6, we prove the assertion in the case $r=1$.

Assume that $K_{R}+\mathscr{D}(E)$ is a Cartier divisor of $R$. Since $\mathscr{D}(E)^{\prime}=\mathscr{D}(E)$, we have $\mathscr{D}(E)=0$ (and hence $E=0$ ). We employ the notation used in Step 1 of the proof of Theorem 2.6. By assumption, $\varphi \in k(X)$ with $K_{X}+D^{\prime}=\operatorname{div}_{X}(\varphi)$. Let $\Psi: C(X, D) \rightarrow \operatorname{Spec}(R)$ be the graded blowing-up as described in 1.1. By setting $\omega_{0}=1 / \varphi$, we have $K_{S}=\omega_{0} S$, and we can show the relation

$$
\omega_{C}=\bigoplus_{k \geq 1} O_{X}\left(K_{X}+D^{\prime}+k D\right) T^{k}=\Psi^{*}\left(\omega_{0}\right) O_{C}(-X) .
$$

Let $\tau: \tilde{C} \rightarrow C$ be a resolution of singularities of $C$, where $\tau^{-1}(X+\operatorname{Sing}(C))$ is a simple normal crossing divisor. We denote the proper transform of $X$ as $\tilde{X} \subset \tilde{C}$. Let us represent the canonical divisor $\operatorname{div}_{\tilde{C}}\left((\Psi \tau)^{*}\left(\omega_{0}\right)\right)$ as $K_{\tilde{C}}=-G_{J}+$ $G_{I}$, where $G_{J}$ and $G_{I}$ are effective divisors on $\tilde{C}$ whose supports have no common irreducible components. Further, since $\operatorname{Spec}(R)-V\left(R_{+}\right)$has only rational singularities, the support of $G_{J}$ is contained in $\tau^{-1}(X)$. It is then clear that $G_{J} \geq \tilde{X}$.

We show the relation $G_{J}=\tilde{X}$ by contradiction. Assume $G_{J} \neq \tilde{X}$. Then $G_{J}-\tilde{X}$ is a non-zero effective divisor. Hence $0 \neq O_{G_{J}-\tilde{X}} \subset O_{G_{J}-\tilde{X}}\left(G_{I}\right)$. We have the natural inclusion relations

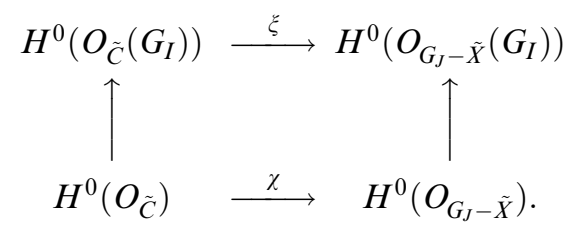

Since $\chi(1) \neq 0, \xi$ is not the zero-map. We have the commutative diagram of exact sequences

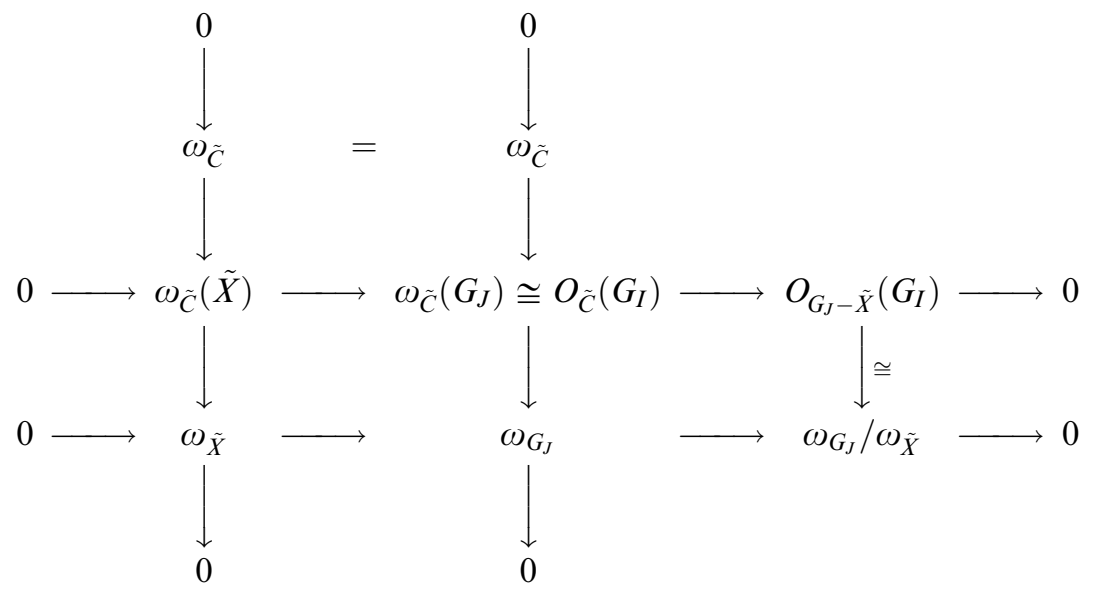


and we have

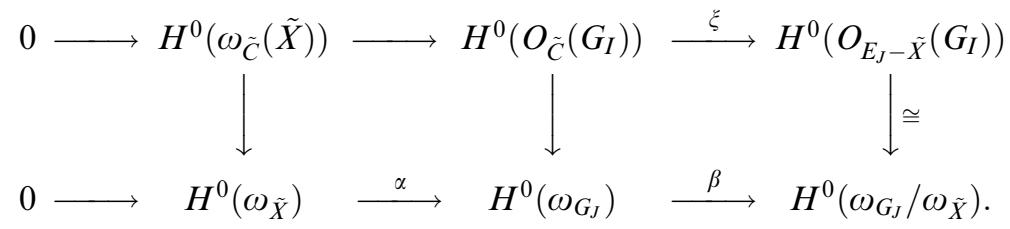

Hence $\beta$ is not the zero-map, and $\alpha$ is not an isomorphism.

In the resolution of singularities $\left.\tau\right|_{\tilde{X}}: \tilde{X} \rightarrow X, X$ has only rational singularities. Hence we have the relation $H^{0}\left(\omega_{\tilde{X}}\right) \cong H^{0}\left(\omega_{X}\right)$. By the GrauertRiemenshneider vanishing theorem we have $H^{1}\left(\tilde{C}, \omega_{\tilde{C}}\right)=0$, and therefore we obtain the exact sequence

$$
0 \rightarrow H^{0}\left(\tilde{C}, \omega_{\tilde{C}}\right) \rightarrow H^{0}\left(\tilde{C}, \omega_{\tilde{C}}\left(G_{J}\right)\right) \rightarrow H^{0}\left(\omega_{G_{J}}\right) \rightarrow 0 .
$$

Since the support of $G_{J}$ is contained in $\varphi^{-1}(X)$, the relation $H^{0}\left(\tilde{C}, \omega_{\tilde{C}}\left(E_{J}\right)\right) \subset$ $H^{0}\left(\tilde{C}-\varphi^{-1}(X), \omega_{\tilde{C}}\right)$ follows. Then, since $C$ has only rational singularities, we obtain

$$
H^{0}\left(\tilde{C}, \omega_{\tilde{C}}\right) \cong H^{0}\left(C, \omega_{C}\right) \text { and } H^{0}\left(\tilde{C}-\varphi^{-1}(X), \omega_{\tilde{C}}\right) \cong H^{0}\left(C-X, \omega_{C}\right) .
$$

Therefore

$$
H^{0}\left(\omega_{G_{J}}\right) \subset \frac{H^{0}\left(C-X, \omega_{C}\right)}{H^{0}\left(C, \omega_{C}\right)} \cong \bigoplus_{k \leq 0} H^{0}\left(O_{X}\left(K_{X}+D^{\prime}+k D\right)\right) T^{k}=H^{0}\left(\omega_{X}\right),
$$

since $K_{X}+D^{\prime}=\operatorname{div}(\varphi)$. However, this contradicts the fact that $\alpha$ is not an isomorphism.

The arguments for the case of general $r$ are the same as those in the proof of Theorem 2.6.

Now, from Theorems 2.7 and 2.8 we obtain the following.

COROLlaRY 2.9. Let $R=R(X, D)$ be a normal graded ring over a field $k$ with $\operatorname{char}(k)=0$, and let $\mathscr{D}(E)$ be a homogeneous $\boldsymbol{Q}$-divisor with $E \in \operatorname{Div}(X) \otimes \boldsymbol{Q}$ such that $\mathscr{D}(E)=\mathscr{D}(E)^{\prime}$. Suppose the following condition holds.

$\left(^{*}\right)$ At each point $x$ of $X,(X, x)$ is klt with respect to $K_{X, x}+D_{x}^{\prime}+E_{x}$.

Then $R(X, D)$ is a log canonical singularity with respect to $K_{R}+\mathscr{D}(E)$ of index $r$ if and only if there exists $a^{\prime} \in \boldsymbol{Z}$ with $a^{\prime} \leq 0$ such that $r\left(K_{X}+D^{\prime}+E\right)-a^{\prime} D$ is an integral principal divisor on $X$ and $r \in N$ is the minimal integer such that this holds.

\section{§3. Cyclic covers of the Kummer type and Veronese subrings}

3.1. Let $S=R(X, D)$ be a normal graded domain with $D=$ $\sum_{V \in \operatorname{Irr}^{1}(X)}\left(p_{V} / q_{V}\right) V$, as in 1.1. For a positive integer $m$, we have the 
natural isomorphism of the $m$ th Veronese subring $S^{(m)} \cong R(X, m D)$. We can reconstruct $S$ as a graded cyclic cover of $S^{(m)}$ as follows: $R(X, D) \cong$ $S\left(R(X, m D), \mathscr{D}_{R(X, m D)}(D), T^{m}\right)$. This is a very natural construction of a cyclic cover. Another familiar method to construct a cyclic cover is that for a cyclic cover of the Kummer type, $S=R[X] /\left(X^{m}-v\right)$ with $v \in R$. Our interest in this section is with the relations between these two types of cyclic covers. Our results in the followings extend S. Mori's structure theorems [Theorems 3.9 and 3.11] regarding graded factorial domains. First, we show the following as a general result concerning of normal Kummer extensions for not necessarily graded cases.

THEOREM 3.2. Let $R$ be a normal domain. For non-units $v_{1}, \ldots v_{n} \in R$ and integers $m_{1}, \ldots, m_{n} \in \boldsymbol{Z}$ with $m_{i} \geq 2$, we define the ring $S$ as $S=R\left[X_{1}, \ldots, X_{n}\right] /$ $\left(X_{1}^{m_{1}}-v_{1}, \ldots, X_{n}^{m_{n}}-v_{n}\right)$. Then the following hold:

(i) $S$ is a normal domain if and only if all $v_{i} R$ are reduced and no pair $v_{i} R$ and $v_{j} R$ with $i \neq j$ has a common prime component.

(ii) $S$ is a cyclic cover of $R$ if and only if $\left(m_{i}, m_{j}\right)=1$ for any $i \neq j$.

Proof. (i) Suppose that $S$ is a normal domain. Then, for any $i, R\left[X_{i}\right] /$ $\left(X_{i}^{m_{i}}-v_{i}\right)$ is a direct summand of $S$, hence is normal by the purerity. In particular, we obtain the isomorphism $S\left(R,\left(1 / m_{i}\right) \operatorname{div}\left(v_{i}\right), v_{i}\right)=R\left[X_{i}\right] /\left(X_{i}^{m_{i}}-v_{i}\right)$. Thus we conclude that $v_{i}$ is reduced. For any pair $i, j$ with $i \neq j, R\left[X_{i}, X_{j}\right] /$ $\left(X_{i}^{m_{i}}-v_{i}, X_{j}^{m_{j}}-v_{j}\right)$ is normal by the same reason as above. At this point, we can conclude that $v_{j}\left(R\left[X_{i}\right] /\left(X_{i}^{m_{i}}-v_{i}\right)\right)$ defines a reduced ideal of $R\left[X_{i}\right] /\left(X_{i}^{m_{i}}-v_{i}\right)$ by the normality of $\left(R\left[X_{i}\right] /\left(X_{i}^{m_{i}}-v_{i}\right)\right)\left[X_{j}\right] /\left(X_{j}^{m_{j}}-v_{j}\right)$. Therefore $R \subset R\left[X_{i}\right]\left(X_{i}^{m_{i}}-v_{i}\right)$ has no ramification at any prime component of $v_{j} R$. In particular, $v_{i} R$ and $v_{j} R$ have no common prime component.

Conversely, suppose that all $v_{i} R$ are reduced and that each pair $v_{i} R$ and $v_{j} R$ have no common prime component for any $i \neq j$. Then we have the relation $S\left(R,\left(1 / m_{1}\right) \operatorname{div}\left(v_{1}\right), v_{1}\right)=R\left[X_{1}\right] /\left(X_{1}^{m_{1}}-v_{1}\right)$, and this is normal (cf. [18] for precise arguments about the normality of such covers). Let for $j \geq 2$. Since $R \subset R\left[X_{1}\right]\left(X_{1}^{m_{1}}-v_{1}\right)$ has no ramification at any prime component of $v_{j} R$, $v_{j} R\left[X_{1}\right]\left(X_{1}^{m_{1}}-v_{1}\right)$ is reduced. Further, we can easily see that $v_{j} R\left[X_{1}\right]\left(X_{1}^{m_{1}}-v_{1}\right)$ and $v_{k} R\left[X_{1}\right]\left(X_{1}^{m_{1}}-v_{1}\right)$ have no common component for $j \neq k$. Hence the normality of $S$ follows by induction on $n$.

The equivalence assertion (ii) is known.

Q.E.D.

Theorem 3.3. Let $R=R(X, D)$ be a normal domain as described in 1.1, let $v_{1}, \ldots v_{n} \in R$ be homogeneous reduced non-units, and let $m_{1}, \ldots, m_{n} \in \boldsymbol{Z}$ with $m_{i} \geq 2$. Here we assume that no pair $v_{i} R$ and $v_{j} R$ has a common prime component and that $\left(m_{i}, m_{j}\right)=1$ for any $i \neq j$. We define the cyclic $m_{1} \cdots m_{n}$-cover $S$ of $R$ by $S=R\left[X_{1}, \ldots, X_{n}\right] /\left(X_{1}^{m_{1}}-v_{1}, \ldots, X_{n}^{m_{n}}-v_{n}\right)$. Then the following hold:

(i) $S^{\left(m_{1} \cdots m_{n}\right)} \cong R$ if and only if $\left(m_{i}, \operatorname{deg}_{R}\left(v_{i}\right)\right)=1$ for $1 \leq i \leq n$.

(ii) Under the conditions stated in of (i), representing $v_{i} R=R\left(-\mathscr{D}\left(E_{i}\right)\right)$ by $E_{i}=\sum_{k=1}^{r_{i}}\left(1 / q_{V_{i, k}}\right) V_{i, k} \in \operatorname{Div}(X, D), 1 \leq i \leq n$, and with $S=R(X, \tilde{D})$ as given in Theorem 1.3, $\tilde{D}$ can be written 


$$
\tilde{D}=\sum_{i=1}^{n} \sum_{k=1}^{r_{i}} \frac{\tilde{p}_{V_{i, k}}}{m_{i} q_{V_{i, k}}} V_{i, k}+\sum_{V \neq V_{i, j}} \frac{\tilde{p}_{V}}{q_{V}} V
$$

where $\left(\tilde{p}_{V_{i, k}}, m_{i} q_{V_{i, k}}\right)=1$, and $\left(\tilde{p}_{V}, q_{V}\right)=1$ for $V \neq V_{i, k}$.

Proof. Let $Q(R)$ (resp. $Q(S))$ be the quotient field of $R$ (resp. $S$ ). Also, let $l_{i}=\prod_{j \neq i} m_{j}$ for $1 \leq j \leq n$. Then, since $(1, \ldots, 1)$ generates $\boldsymbol{Z} / m_{1} \boldsymbol{Z} \oplus \cdots$ $\oplus \boldsymbol{Z} / m_{n} \boldsymbol{Z}$, defining $\tilde{X} \in Q(S)$ as $\tilde{X}=\prod_{i=1}^{n} X_{i}$, we have the relation $Q(S)=$ $Q(R)[\tilde{X}] /\left(\tilde{X}^{m_{1} \cdots m_{n}}-\prod_{i=1}^{n} v_{i}^{l_{i}}\right)$. As shown in [18], we can represent the cyclic $m_{1} \cdots m_{n}$-cover $S$ of $R$ as follows:

$$
S=S\left(R, \frac{1}{m_{1} \cdots m_{n}} \operatorname{div}_{R}\left(\prod_{i=1}^{n} v_{i}^{l_{i}}\right), \prod_{i=1}^{n} v_{i}^{l_{i}}\right) .
$$

Further, we have the relations

$$
\frac{1}{m_{1} \cdots m_{n}} \operatorname{div}_{R}\left(\prod_{i=1}^{n} v_{i}^{l_{i}}\right)=\mathscr{D}\left(\frac{1}{m_{1} \cdots m_{n}} \sum_{i=1}^{n} l_{i} E_{i}\right)
$$

and

$$
\left(m_{1} \cdots m_{n}\right)\left(\frac{1}{m_{1} \cdots m_{n}} \sum_{i=1}^{n} l_{i} E_{i}\right)-\left(\sum_{i=1}^{n} l_{i} \operatorname{deg}_{R}\left(v_{i}\right)\right) D \in P(X) .
$$

We can easily see that $\left(\left(m_{1} \cdots m_{n}\right), \sum_{i=1}^{n} l_{i} \operatorname{deg}_{R}\left(v_{i}\right)\right)=\prod_{i=1}^{n}\left(m_{i}, \operatorname{deg}_{R}\left(v_{i}\right)\right)$. Therefore the assertion (i) follows from assertion (2) of Remark 1.4.

(ii) We have

$$
\frac{1}{m_{1} \cdots m_{n}} \sum_{i=1}^{n} l_{i} E_{i}=\sum_{i=1}^{n} \frac{1}{m_{i}} E_{i}=\sum_{i=1}^{n} \sum_{k=1}^{r_{i}} \frac{1}{m_{i} q_{V_{i, k}}} V_{i, k} .
$$

Therefore, by Theorem 1.5 and Remark 1.6, the ramification of $\tilde{D}$ at $V_{i, k}$ is $m_{i} q_{V_{i, k}}$ and at $V \neq V_{i, k}$ is $q_{V}$.

Q.E.D.

The following is the converse of Theorem 3.3.

THEOREM 3.4. Let $S=R(X, D)$ be a normal domain as described in 1.1, and let $m>1$ be an integer. We consider the following conditions.

(a) If $\left(m, q_{V}\right) \neq\left(m, q_{V^{\prime}}\right)$ then $\left(\left(m, q_{V}\right),\left(m, q_{V^{\prime}}\right)\right)=1$. Further, if $m_{1}, \ldots, m_{n}$ are the possible different values of each $\left(m, q_{V}\right)$ with $m_{i} \geq 2$, then $m=\prod_{i=1}^{n} m_{i}$ holds.

(b) For each $m_{i}$, let $V_{i, 1}, \ldots, V_{i, r_{i}}$ be the components such that $\left(m_{i}, q_{V_{i, j}}\right) \geq 2$. Then $E_{i}=\sum_{k=1}^{r_{i}}\left(1 / q_{V_{i, k}}\right) V_{i, k}$ defines a principal divisor $S\left(-\mathscr{D}_{S}\left(E_{i}\right)\right)=t_{i} S$. Further, for any $i \neq j$, we have $\left\{V_{i, 1}, \ldots, V_{i, r_{i}}\right\} \cap\left\{V_{j, 1}, \ldots, V_{j, r_{j}}\right\}=\emptyset$.

Assume conditions (a) and (b) hold. Then (i) $t_{i}^{m_{i}}$ is a reduced element of $S^{(m)}$ 
with $\left(m_{i}, \operatorname{deg}_{S^{(m)}}\left(t_{i}^{m_{i}}\right)\right)=1$ for $1 \leq i \leq n$, (ii) no pair $t_{i}^{m_{i}} S^{(m)}$ and $t_{j}^{m_{j}} S^{(m)}$ has a common prime component for $i \neq j$, and (iii) we have the isomorphism

$$
S \cong S^{(m)}\left[X_{1}, \ldots, X_{n}\right] /\left(X_{1}^{m_{1}}-t_{1}^{m_{i}}, \ldots, X_{n}^{m_{n}}-t_{n}^{m_{n}}\right) .
$$

Proof. By assumption there exist $a_{i}^{\prime} \in \boldsymbol{Z}$ and $\varphi_{i} \in k(X)$ such that

$$
E_{i}-a_{i}^{\prime} D=\operatorname{div}_{X}\left(\varphi_{i}\right), \quad \text { i.e., } t_{i}=\varphi_{i} T^{a_{i}^{\prime}} \text { for } 1 \leq i \leq n .
$$

Hence $a_{i}^{\prime} p_{V_{i, k}} \equiv 1 \bmod q_{V_{i, k}}$ and $q_{V} \mid a_{i}^{\prime}$ for $V \notin\left\{V_{i, 1}, \ldots, V_{i, r_{i}}\right\}$. We thus obtain the relations $\left(a_{i}^{\prime}, q_{V_{i, k}}\right)=1$ and $a_{i}^{\prime} /\left(\prod_{j \neq i} m_{j}\right) \in \boldsymbol{Z}$. Hence

$$
m_{i} E_{i}-\frac{a_{i}^{\prime}}{\prod_{j \neq i} m_{j}}(m D)=\operatorname{div}_{X}\left(\varphi_{i}^{m_{i}}\right) \quad \text { for } 1 \leq i \leq n \text {. }
$$

It can then be shown that $t_{i}^{m_{i}} \in\left(S^{(m)}\right)_{a_{i}^{\prime} /\left(\prod_{j \neq i} m_{j}\right)}$ and $\left(m_{i}, a_{i}^{\prime} /\left(\prod_{j \neq i} m_{j}\right)\right)=1$. Further, we have

$$
m D=\sum_{i=1}^{n} \sum_{k=1}^{r_{i}} \frac{\left(\prod_{j \neq i} m_{j}\right) p_{V_{i, k}}}{q_{V_{i, k}} / m_{i}} V_{i, k}+\sum_{V \neq V_{i, k}} \frac{m p_{V}}{q_{V}} V
$$

with $\left(\left(\prod_{j \neq i} m_{j}\right) p_{V_{i, k}}, q_{V_{i, k}} / m_{i}\right)=1$ and $\left(m p_{V}, q_{V}\right)=1$ for $V \neq V_{i, k}$. Hence $m_{i} E_{i}=$ $\sum_{k=1}^{r_{i}}\left(1 /\left(q_{V_{i, k}} / m_{i}\right)\right) V_{i, k}$ defines a reduced divisor of $S^{(m)} \cong R(X, m D)$. Therefore the assertions of (i) and (ii) follow.

Since (i) and (ii) hold, if we define $\tilde{S}$ by

$$
\tilde{S}=S^{(m)}\left[X_{1}, \ldots, X_{n}\right] /\left(X_{1}^{m_{1}}-t_{1}^{m_{1}}, \ldots, X_{n}^{m_{n}}-t_{n}^{m_{n}}\right),
$$

then, by the similar arguments in the proof of Theorem 3.3, we can show the relation

$$
\left.\tilde{S}=S\left(R(X, m D), \mathscr{D}_{R(X, m D)}\left(\sum_{i=1}^{n} E_{i}\right),\left(t_{1} \cdots t_{n}\right)^{m}\right)\right) .
$$

Here we have $m \sum_{i=1}^{n} E_{i}-\left(\sum_{i}^{n} a_{i}^{\prime}\right)(m D)=m \operatorname{div}_{X}\left(\prod_{i=1}^{n} \varphi_{i}\right)$ and $\left(m, \sum_{i}^{n} a_{i}^{\prime}\right)=1$. Now, let $\alpha, \beta \in \boldsymbol{Z}$ such that $\alpha\left(\sum_{i}^{n} a_{i}^{\prime}\right)+\beta m=1$. Then defining $\tilde{D}$ as $\tilde{D}=$ $\alpha\left(\sum_{i=1}^{n} E_{i}\right)+\beta(m D)$, we have $\tilde{S} \cong R(X, \tilde{D})$. Hence $\tilde{D}-D=\alpha\left(\sum_{i=1}^{n} E_{i}\right)+$ $\beta(m D)-\left(\alpha\left(\sum_{i}^{n} a_{i}^{\prime}\right)+\beta m\right) D=\sum_{i=1}^{n} \alpha\left(E_{i}-a_{i}^{\prime} D\right)=\sum_{i=1}^{n} \operatorname{div}\left(\varphi_{i}^{\alpha}\right)$. Therefore $S \cong$ $S^{(m)}\left[X_{1}, \ldots, X_{n}\right] /\left(X_{1}^{m_{1}}-t_{1}^{m_{1}}, \ldots, X_{n}^{m_{n}}-t_{n}^{m_{n}}\right)$.

Q.E.D.

Example 3.5. (i) Let $R$ be a normal graded ring and $R=R_{0}\left[x_{1}, \ldots, x_{s}\right]$ with $x_{1}, \ldots, x_{s}$ a minimal homogeneous generator of $R_{+}=\oplus_{k \geq 1} R_{k}$. Also, with $a_{i}=\operatorname{deg}\left(x_{i}\right)$ for $i=1, \ldots, s$, we assume $\left(a_{1}, \ldots, a_{s}\right)=1$. Next we define $c_{i}$ by $c_{i}=\left(a_{1}, \ldots, a_{i-1}, a_{i+1}, \ldots, a_{x}\right)$ for $i=1, \ldots, s$ and write $L=\prod_{1 \leq i \leq s} c_{i}$. Then it is wellknown that $R_{0}\left[x_{1}, \ldots, x_{s}\right]^{(L)}=R_{0}\left[x_{1}^{\prime}, \ldots, x_{s}^{\prime}\right]$, where $x_{i}^{\prime}=x_{i}^{c_{i}}$ for $i=1, \ldots, s$. Hence we obtain the relation

$$
R \cong R^{(L)}\left[x_{1}, \ldots, x_{s}\right] /\left(x_{1}^{c_{1}}-x_{1}^{\prime}, \ldots, x_{s}^{c_{s}}-x_{s}^{\prime}\right) .
$$


By this relation, we can obtain the following:

(3.5.1) If $c_{i} \geq 2$ for some $i$, then $x_{i} R$ and $x_{i}^{\prime} R^{(L)}$ are reduced.

(3.5.2) If $c_{i} \geq 2$ and $c_{j} \geq 2$ for some pair $i, j$, with $i \neq j$, then $x_{i} R$ and $x_{j} R$ (resp. $x_{i}^{\prime} R^{(L)}$ and $x_{j}^{\prime} R^{(L)}$ ) do not have a common prime component by Theorems 3.3 and 3.4. Hence $\operatorname{ht}\left(x_{i}, x_{j}\right) R \geq 2\left(\operatorname{resp} . \operatorname{ht}\left(x_{i}^{\prime}, x_{j}^{\prime}\right) R^{(L)} \geq 2\right)$.

(3.5.3) Let $R=R(X, D)$ as in 1.1. Assume that $c_{i} \geq 2$ for $i=1, \ldots, s$. Then, by the arguments above, we can see that $q_{V}=c_{i}$ at the components $V \subset V\left(x_{i} R\right)$ and $L D$ is an integral divisor.

(ii) Let us next consider the case $R=k\left[x_{1}, x_{2}, x_{3}\right] / f$, where $f$ is a weighted homogeneous polynomial of degree $h$ and $k$ is an algebraically closed field with $\operatorname{char}(k)=0$. In Assertion 1.6.4 of [14], K. Saito showed that $L \mid h$. Hence there exists $g \in k\left[x_{1}^{\prime}, x_{2}^{\prime}, x_{3}^{\prime}\right]$ with $g\left(x_{1}^{\left(a_{2}, a_{3}\right)}, x_{2}^{\left(a_{1}, a_{3}\right)}, x_{3}^{\left(a_{1}, a_{2}\right)}\right)=f\left(x_{1}, x_{2}, x_{3}\right)$ and $R^{(L)}=$ $k\left[x_{1}^{\prime}, x_{2}^{\prime}, x_{3}^{\prime}\right] / g$. We can see that the denominators $q_{V}$ of the fractional parts of $D$ have at most 4 types, by arguments similar to those in (i).

In the rest of this section, we discuss the class groups. First, we show the following.

THeOREM 3.6. Let $S=R(X, D)$ as in 1.1 and let $S^{(m)} \cong R(X, m D)$. Then the natural map $\alpha: \mathrm{Cl}\left(S^{(m)}\right) \rightarrow \mathrm{Cl}(S)$ is an isomorphism if and only if $m=\prod\left(m, q_{V}\right)$ and $\left(\left(m, q_{V}\right),\left(m, q_{V^{\prime}}\right)\right)=1$ for any $V \neq V^{\prime}$.

Proof. By Proposition 1.9, we have the exact sequence

$$
0 \rightarrow \boldsymbol{Z} \operatorname{cl}\left(L \mathscr{D}_{R(X, m D)}(D)\right) \rightarrow \mathrm{Cl}(R(X, m D)) \rightarrow \mathrm{Cl}(R(X, D)) \rightarrow \text { Coker } \beta \rightarrow 0,
$$

where $L$ and $\beta$ are defined as in 1.8. Also, we have $m D=\sum_{V \in \operatorname{Irr}^{1}(X)}$ $\left(\left(m p_{V} /\left(m, q_{V}\right)\right) /\left(q_{V} /\left(m, q_{V}\right)\right)\right) V$, with $\left(m p_{V} /\left(m, q_{V}\right), q_{V} /\left(m, q_{V}\right)\right)=1$ for all $V$. Then we have the representation $\mathscr{D}_{R(X, m D)}(D)=\sum_{V \in \operatorname{Irr}^{1}(X)}\left(p_{V} /\left(m, q_{V}\right)\right) \mathscr{V}$, where $\mathscr{V} \in \operatorname{Div}(R(X, m D))$ is the prime divisor associated with $V \in \operatorname{Irr}^{1}(X)$. Hence $L=\operatorname{LCM}\left\{\left(m, q_{V}\right)\right\}$ and $\beta: \boldsymbol{Z} \rightarrow \bigoplus_{V \in \operatorname{Irr}^{1}(X)} \boldsymbol{Z} /\left(m, q_{V}\right) \boldsymbol{Z}$ by $\beta(1)=\left(p_{V}\right)$. Now $\operatorname{cl}\left(L \mathscr{D}_{R(X, m D)}(D)\right)=0$ if and only if $L=m$. Further, it is easy to see that $\operatorname{Coker}(\beta)=0$ if and only if $L=\prod\left(m, q_{V}\right)$.

Q.E.D.

Combining Theorem 3.6 with Theorem 3.4, we obtain the following.

Corollary 3.7. Let $S=R(X, D)$ be as in 1.1 and let $m>0$ be an integer. Suppose that the natural map $\alpha: \mathrm{Cl}\left(S^{(m)}\right) \rightarrow \mathrm{Cl}(S)$ is an isomorphism and that $S\left(-\left(1 / q_{V}\right) V\right) \subset S$ is a principal prime ideal for any $V \in \operatorname{Irr}^{1}(X)$ with $\left(m, q_{V}\right) \geq 2$. Then $S$ is a graded cyclic cover of $S^{(m)}$ obtained by the Kummer extension with the following conditions, in which $V_{1}, \ldots, V_{n}$ are the prime divisors with $m_{V} \geq 2$.

(i) $\left(m, q_{V_{i}}\right)$ and $\left(m, q_{V_{j}}\right)$ are relatively prime for any $i, j$ with $i \neq j$ and the equality $m=\prod\left(m, q_{V}\right)$ holds. We set $m_{i}=\left(m, q_{V_{i}}\right)$.

(ii) There is a homogeneous prime element $v_{i}$ of $S^{(m)}$ such that $S^{\left(m_{i}\right)}\left(-\left(m_{i} / q_{V_{i}}\right) V_{i}\right)=v_{i} S^{\left(m_{i}\right)},\left(m_{i}, \operatorname{deg}_{S^{(m)}}\left(v_{i}\right)\right)=1$ for $i=1, \ldots, n$, and 


$$
S \cong S^{(m)}\left[X_{1}, \ldots, X_{n}\right] /\left(X_{1}^{m_{1}}-v_{1}, \ldots, X_{n}^{m_{n}}-v_{n}\right) .
$$

We will apply this to a graded UFD. First, we recall the following.

Theorem 3.8 [20]. Let $R=R(X, D)$ as in 1.1. Then $\mathrm{Cl}(R)=0$ if and only if $\left(q_{V}, q_{V^{\prime}}\right)=1$ for any pair $V \neq V^{\prime}$ and $Z \mathrm{cl}(L D)=\mathrm{Cl}(X)$, where $L=$ $\operatorname{LCM}\left(q_{V}\right)=\prod_{V \in \operatorname{Irr}^{1}(X)} q_{V}$.

As a corollary we can show the following. Then

Theorem 3.9 (S. Mori [10]). Let $R=R(X, D)$ be a UFD as described above.

$$
R \cong R(X, L D)\left[u_{1}, \ldots, u_{m}\right] /\left(u_{1}^{q_{V_{1}}}-v_{V_{1}}, \ldots, u_{m}^{q_{V_{m}}}-v_{V_{m}}\right),
$$

where $v_{V_{i}} \in R(X, L D)_{e_{i}}$ is a prime element such that $\left(q_{V_{i}}, e_{i}\right)=1$ for $i=1, \ldots, m$.

Proof. By Theorem 3.8, we can see $\mathrm{Cl}(R(X, L D))=0$. Further, for any $V \in \operatorname{Irr}^{1}(X), \quad\left(1 / q_{V}\right) V \in \operatorname{Div}(X, D)=\boldsymbol{Z} D \oplus P(X)$; i.e., there are $a_{V}^{\prime} \in \boldsymbol{Z}$ and $\varphi_{V} \in k(X)$ such that $\left(1 / q_{V}\right) V-a_{V}^{\prime} D=\operatorname{div}\left(\varphi_{V}\right)$. Hence we obtain the assertion by Corollary 3.7. Here we can see $e_{i}=q_{V_{i}} a_{i}^{\prime} / L$.

Q.E.D.

Next we study the cyclic cover of the Kummer type. As a corollary of Theorems 3.3 and 3.6, we obtain the following.

THEOREM 3.10. Let $R=R(X, D)$ be as in 1.1 , and let $v_{i} \in R$ be homogeneous prime elements for $i=1, \ldots, n$. Also, let $m_{1}, \ldots, m_{n}$ be positive integers such that $\left(m_{i}, \operatorname{deg}\left(v_{i}\right)\right)=1$ for any $i$ and $\left(m_{i}, m_{j}\right)=1$ for $i \neq j$. Then

$$
S=R\left[X_{1}, \ldots, X_{n}\right] /\left(X_{1}^{m_{1}}-v_{1}, \ldots, X_{n}^{m_{n}}-v_{n}\right)
$$

is a normal graded cyclic cover of $R$ with $R=S^{\left(m_{1} \cdots m_{n}\right)}$ and the natural map $\mathrm{Cl}(R) \rightarrow \mathrm{Cl}(S)$ is isomorphic.

COROLlary 3.11 (cf. [10]). Let $R=R(X, D)$ be a graded UFD as in 1.1, and let $v_{i} \in R$ be homogeneous prime elements for $i=1, \ldots, n$. Also, let $m_{1}, \ldots, m_{n}$ be positive integers such that $\left(m_{i}, \operatorname{deg}\left(v_{i}\right)\right)=1$ for any $i$ and $\left(m_{i}, m_{j}\right)=1$ for $i \neq j$. Then

$$
S=R\left[X_{1}, \ldots, X_{n}\right] /\left(X_{1}^{m_{1}}-v_{1}, \ldots, X_{n}^{m_{n}}-v_{n}\right)
$$

is a UFD with $R=S^{\left(m_{1} \cdots m_{n}\right)}$.

\section{§4. A few remarks on integral cyclic covers}

We now give some remarks on integral cyclic covers. By using Flenner's result, in the characteristic zero case we can show the following, which basically asserts that every integral cyclic cover of the completion of a graded ring is graded cyclic cover. 
Proposition 4.1. Let $R=R(X, D)$ be as in 1.1 , let $r>0$ an integer, and let $R^{\wedge}$ be the completion of $R$. We assume that $R_{0}=k$ is an algebraically closed field of characteristic zero. Let $\pi: Z \rightarrow \operatorname{Spec}\left(R^{\wedge}\right)$ be an integral r-cyclic cover. Then there exits $E \in \operatorname{Div}(X, D)$ and $r E-a^{\prime} D=\operatorname{div}(\varphi)$ as in 1.2 such that $\pi$ is obtained from the graded integral r-cyclic cover $R \subset S=S\left(R, \mathscr{D}(E), \varphi T^{a^{\prime}}\right)$ by the completion.

Proof. By assumption we can choose $\mathscr{D} \in \operatorname{Div}\left(R^{\wedge}\right)$ which defines a torsion of $\mathrm{Cl}\left(R^{\wedge}\right)$ of order $r$ and obtain the relation $S\left(R^{\wedge}, \mathscr{D}, \Phi\right) \cong O_{Z}$, where $\Phi$ is an element of the quotient field of $R^{\wedge}$ with $r \mathscr{D}=\operatorname{div}(\Phi)$. As demonstrated in $\S 4$ of [4], the cokernel of the injection $\mathrm{Cl}(R) \rightarrow \mathrm{Cl}\left(R^{\wedge}\right)$ is torsion free. Hence we can choose $E \in \operatorname{Div}(X, D)$ such that $\operatorname{cl}(\mathscr{D}(E))=\operatorname{cl}(\mathscr{D})$ in $\operatorname{Cl}\left(R^{\wedge}\right)$. Therefore we obtain an isomorphism $S\left(R^{\wedge}, \mathscr{D}(E), \varphi T^{a^{\prime}}\right) \cong S\left(R^{\wedge}, \mathscr{D}, \Phi\right)$, since we can take the $r$ th root of units of $R^{\wedge}$ (see the remark following Example 1.6 of [18]).

Q.E.D.

(4.2) As is seen in 1.2, our cyclic cover $S=S\left(R, \mathscr{D}(E), \varphi T^{a^{\prime}}\right)$ possesses a natural bigraded structure corresponding to the divisor class. This fact is very useful for obtaining an explicit representation of the $\boldsymbol{Z} / r \boldsymbol{Z}$-action on $S$. For a standard generator $\zeta \in \boldsymbol{Z} / r \boldsymbol{Z}$, we have $\zeta^{*}\left(\varphi T^{i} u^{j}\right)=\zeta^{j}\left(\varphi T^{i} u^{j}\right)$. Since $S_{n}=\bigoplus_{l=0}^{s} S_{n, l}$ with $S_{n, l}=H^{0}\left(X, O_{X}\left(l\left((r / s) E-\left(a^{\prime} / s\right) D\right)+n(\alpha E+\beta D)\right) T^{-\left(l a^{\prime} / s\right)+n \beta} u^{(l r / s)+n \alpha}\right.$. Hence we obtain the relation $\zeta^{*}(x)=\zeta^{(r / s)+n \alpha} x$ for $x \in S_{n, l}$.

Cyclic covers of rational double points are wellknown and important in studies of terminal singularities [13]. With Proposition 4.1, their study can be reduced to that of class groups. Here we will give a simple example.

Example $4.3\left(D_{2 m+2} \rightarrow D_{4 m+2}\right)$. Let $R=R\left(\boldsymbol{P}^{1}, D\right)$ be a rational double point of type $D_{n}$ with $n=4 m+2$ and $m \in \boldsymbol{Z}_{>0}$, where $D=(1 / 2) P_{1}+(1 / 2) P_{2}-$ $((n-3) /(n-2)) P_{3}$ and $P_{1}, P_{2}, P_{3}$ are three distinct points of $\boldsymbol{P}^{1}$. We have $\mathrm{Cl}(R)=\left\{0, \operatorname{cl}\left((1 / 2) P_{1}\right), \operatorname{cl}\left((1 / 2) \mathrm{P}_{2}\right), \operatorname{cl}\left((1 / 2) P_{1}+(1 / 2) P_{2}\right)\right\} \cong \boldsymbol{Z} / 2 \boldsymbol{Z} \oplus \boldsymbol{Z} / 2 \boldsymbol{Z}$. We study the $\boldsymbol{Z} / r \boldsymbol{Z}$-action on the cyclic cover $S$ associated with $\mathscr{D}\left((1 / 2) P_{1}\right)$. We have $(1 / 2) P_{1}-2 m D=(1 / 2) P_{1}-m P_{1}-m P_{2}+2 m P_{3}-(1 / 2) P_{3} \sim(1 / 2) P_{1}-$ $(1 / 2) P_{3}$. Hence $r=2, a^{\prime}=4 m$, and $s=2$. Now, we can choose $(\alpha, \beta)=(0,1)$ and define $\tilde{D}=\rho^{*}(D)=P_{1}^{*}+(1 / 2)\left\{P_{2,1}^{*}+P_{2,2}^{*}\right\}-((4 m-1) / 2 m) P_{3}^{*}$, where $\rho$ : $Y \rightarrow X$ with $\rho^{*}\left\{P_{1}\right\}=\left\{P_{1}^{*}\right\}, \rho^{*}\left\{P_{2}\right\}=\left\{P_{2,1}^{*}, P_{2,2}^{*}\right\}$, and $\rho^{*}\left\{P_{3}\right\}=\left\{P_{3}^{*}\right\}$. Hence $S=R(Y, \tilde{D})$ is a rational double point of type $D_{2 m+2}$. We take $x_{1}, x_{2}$, and $x_{3}$ so as to satisfy $k x_{1}=S_{2,0}=H^{0}\left(\boldsymbol{P}^{1}, O_{\boldsymbol{P}^{1}}\left(P_{1}+P_{2}-2 P_{3}\right)\right) T^{2}, \quad k x_{2}=S_{2 m, 1}=$ $H^{0}\left(\boldsymbol{P}^{1}, O_{\boldsymbol{P}^{1}}\right) u$, and $k x_{3}=S_{2 m+1,1}=H^{0}\left(\boldsymbol{P}^{1}, O_{\boldsymbol{P}^{1}}\left(P_{1}-P_{3}\right)\right) T u$. Then, there is a linear relation among $x_{1}^{2 m+1}, x_{1} x_{2}^{2}$, and $x_{3}^{2}$ in $S_{4 m+2,0}=H^{0}\left(\boldsymbol{P}^{1}, O_{\boldsymbol{P}^{1}}\left((2 m+1) P_{1}+\right.\right.$ $\left.\left.(2 m+1)-(4 m+1) P_{3}\right)\right) T^{4 m+2} \cong k^{2}$. It is easily seen that $S \cong k\left[x_{1}, x_{2}, x_{3}\right] /$ $\left(x_{3}^{2}+x_{1}^{2 m+1}+x_{1} x_{2}^{2}\right)$. Thus, the action of $\boldsymbol{Z} / 2 \boldsymbol{Z}$ is given by $\left(x_{1}, x_{2}, x_{3}\right) \rightarrow$ $\left(x_{1},-x_{2},-x_{3}\right)$.

Index one covers (or canonical covers) of 2-dimensional rational singularities have been studied from several points of view by many authors. However, in 
general, the resulting Gorenstein singularities have invariants that are much different from those of Gorenstein rational singularities. We close this paper by showing following.

Proposition 4.4. Let $R$ be the rational triple point given by $R=R\left(\boldsymbol{P}^{1}, D\right)$ with $D=(1 / 2 a) P_{1}+(1 /(2 a+1)) P_{2}+(1 /(2 a(2 a+1))) P_{3}$, where $P_{1}, P_{2}$, and $P_{3}$ are distinct points of $\boldsymbol{P}^{1}$ and $a \geq 1$. Then the numerical invariants of the index one cover $S=R(Y, \tilde{D})$ of $R$ are as follows: the index of $R$ is $2(2 a+1), a(S)=$ $a-1$, the genus of $Y$ is $a, p_{g}(S)=(1 / 2) a(a+1)$, and $\operatorname{embdim}(\tilde{R})_{\tilde{R}_{+}} \geq 2 a+4$.

Proof. Set $K_{P^{1}}=-2 P_{1}$. Then, we have $K_{P^{1}}+D^{\prime}-(a-1) D \sim(1 / 2) P_{1}-$ $(a /(2 a+1)) P_{2}-(1 /(2(2 a+1))) P_{3}$. Hence the index $r$ of $R$ is $2(2 a+1)$. We thus obtain $a^{\prime}=2(2 a+1)(a-1), s=\left(r, a^{\prime}\right)=2(2 a+1)$, and $a(\tilde{R})=a^{\prime} / s=$ $a-1$. Let us consider $Y=\operatorname{Spec}_{\boldsymbol{P}^{1}}\left(\bigoplus_{l=0}^{s-1} O_{\boldsymbol{P}^{1}}\left((l / 2) P_{1}-(l a /(2 a+1)) P_{2}-\right.\right.$ $\left.\left.(l /(2(2 a+1))) P_{3}\right)\right) \stackrel{\rho}{\rightarrow} X=\boldsymbol{P}^{1}$. Here $\rho$ is a cyclic cover of degree $s=2(2 a+1)$ with branching points $P_{1}, P_{2}$ and $P_{3}$. Here we make the following definitions: $\rho^{-1}\left(P_{1}\right)=\left\{P_{1, i}^{*} ; i=1, \ldots, 2 a+1\right\}, \rho^{-1}\left(P_{2}\right)=\left\{P_{2,1}^{*}, P_{2,2}^{*}\right\}$, and $\rho^{-1}\left(P_{3}\right)=\left\{P_{3}^{*}\right\}$.

By Hurwitz's formula, we have $g(Y)=a$. Further, we can choose $(\alpha, \beta)=(0,1)$. Hence

$$
\tilde{D}=\rho^{*}(D)=\sum_{i=1}^{2 a+1} \frac{1}{a} P_{1, i}^{*}+P_{2,1}^{*}+P_{2,2}^{*}+\frac{1}{a} P_{3}^{*} \sim Q-\sum_{i=1}^{2 a+1} \frac{a-1}{a} P_{1, i}^{*}-\frac{a-1}{a} P_{3}^{*},
$$

where $Q=\sum_{i=1}^{2 a+1} P_{1, i}^{*}+P_{2,1}^{*}+P_{2,2}^{*}+P_{3}^{*}$, with $\operatorname{deg}(Q)=2 a+4$. We regard $S$ as a bigraded ring, as in (4.2): $S_{k, l}=H^{0}\left(\boldsymbol{P}^{1}, O_{\boldsymbol{P}^{1}}\left(F_{l, k}\right)\right) u^{l} T^{n-l(a-1)}$, where

$$
F_{l, k}=\left[l\left(\left(K_{\boldsymbol{P}^{1}}+D^{\prime}\right)-(a-1) D\right)+k D\right]=\left[\frac{l a+k}{2 a}\right] P_{1}+\left[\frac{k-l a}{2 a+1}\right] P_{2}+\left[\frac{k-l a}{2 a(2 a+1)}\right] P_{3}
$$

for integers $l$ and $k$. We study $S_{k}$ with $k \leq a-1=a(S)$. For $l=2 m$, with $0 \leq m \leq 2 a$, we have

$$
\begin{aligned}
& F_{2 m, 0}= \begin{cases}0 & \text { if } m=0, \\
m P_{1}-m P_{2}-P_{3} & \text { if } 0<m \leq 2 a\end{cases} \\
& F_{2 m, k}= \begin{cases}0 & \text { if } m=0, \\
m P_{1}-m P_{2}-P_{3} & \text { if } 0<m \leq 2 a-k, \\
m P_{1}-(m-1) P_{2}-P_{3} & \text { if } 2 a-k+1 \leq m \leq 2 a .\end{cases}
\end{aligned}
$$

For $l=2 m+1$, with $0 \leq m \leq 2 a$, we have

$$
F_{2 m+1, k}= \begin{cases}m P_{1}-(m+1) P_{2}-P_{3} & \text { if } 0 \leq m \leq a-k-1 \\ m P_{1}-m P_{2}-P_{3} & \text { if } a-k \leq m \leq 2 a .\end{cases}
$$

Therefore $p_{g}(S)=\sum_{k=0}^{a(S)} \operatorname{dim} S_{k}=\sum_{k=0}^{a-1}(k+1)=a(a+1) / 2$ (cf. Corollary 2.10 of [21]). 
We next show $\operatorname{embdim}(\tilde{R})_{\tilde{R}_{+}} \geq 2 a+4$. For $k=a$, we have

$$
\begin{gathered}
F_{2 m, a}= \begin{cases}0 & \text { if } m=0, \\
m P_{1}-m P_{2}-P_{3} & \text { if } 1 \leq m \leq a, \\
m P_{1}-(m-1) P_{2}-P_{3} & \text { if } a+1 \leq m \leq 2 a,\end{cases} \\
F_{2 m+1, a}= \begin{cases}P_{1} & \text { if } m=0, \\
(m+1) P_{1}-m P_{2}-P_{3} & \text { if } 1 \leq m \leq 2 a .\end{cases}
\end{gathered}
$$

Here $S_{k, 2 m+1}=0$ for $0 \leq m \leq 2 a$. Hence $\bigoplus_{m=0}^{2 a} S_{a, 2 m+1} \cap\left(\bigoplus_{k \geq 1} S_{k}\right)^{2}=0$, since $s$ is even. Therefore we obtain the relations

$$
\operatorname{embdim}\left(S_{(S)_{+}}\right) \geq \operatorname{dim} S_{1}+\sum_{m=0}^{2 a} \operatorname{dim} S_{a, 2 m+1}=2+2+\sum_{m=1}^{2 a} 1=2 a+4 .
$$

\section{REFERENCES}

[1] M. Demazure, Anneaux gradués normaux, Introduction a la Théorie des Singularités II (Lê Dũng Tráng ed.), Travaux en Cours 37, Hermann, Paris, 1988, 35-68.

[2] R. ElKiK, Rationalité des singularités canoniques, Invent. Math., 64 (1981), 1-6.

[ 3 ] H. FlenNer, Raionale quasihomogene Singularitäten, Arch. Math., 36 (1981), 35-44.

[4] H. FlenNER, Divisorenklassengruppen quasihomogener Singularitäten, J. Reine Angew. Math., 328 (1981), 128-160.

[ 5 ] S. Goto and K.-I. Watanabe, On graded rings I, J. Math. Soc. Japan, 30 (1978), 179-213.

[6] S. ItTAKA, Algebraic Geometry, Graduate Texts in Math. 76, Springer-Verlag, New York, 1981.

[ 7 ] S. IsHII, On isolated Gorenstein singularities, Math. Ann., 270 (1985), 541-554.

[ 8 ] Y. Kawamata, The cone of curves of algebraic varieties, Ann. of Math., 199 (1984), 603633.

[9] J. Kollár, Singularities of pairs, Algebraic Geometry, Santa Cruz 1995 (J. Kollár, R. Lazarsfeld and D. R. Morrison eds.), Proc. Sympos. Pure Math. 62 Part 1, AMS, Providence, 1997, 221-287.

[10] S. MorI, Graded factorial domains, Japan. J. Math., 3 (1977), 223-238.

[11] H. Pinkham, Normal surface singularities with $\boldsymbol{C}^{*}$-action, Math. Ann., 227 (1977), 183-193.

[12] M. ReID, Canonical 3-folds, Journées de Géométrie Algébrique d'Angers, Algebraic Geometry Angers 1979 (A. Beauville ed.), Sijthoff and Noordhoff, Alphen aan den Rijn, 1980, 273-310.

[13] M. ReID, Young person's guide to canonical singularities, Algebraic Geometry, Bowdoin 1985 (S. J. Bloch ed.), Proc. Sympos. Pure Math. 46 Part 1, AMS, Providence, 1987, 354414.

[14] K. SAIto, Regular system of weights and associated singularities, Complex Analytic Singularities (T. Suwa and P. Wagreich eds.), Adv. Stud. Pure Math. 8, Kinokuniya, Tokyo, 1986, 479-526.

[15] P. Samuel, Lectures on Unique Factorization Domains, Tata Inst. Fund. Res. Lectures on Math. and Phys. 30, Tata Inst. Fund. Res., Bombay, 1964.

[16] M. Tomari, The canonical filtration of higher dimensional purely elliptic singularity of a special type, Invent. Math., 104 (1991), 497-520. 
[17] M. Tomari and K.-I. Watanabe, Filtered rings, filtered blowing-ups and normal twodimensional singularities with "star-shaped" resolution, Publ. Res. Inst. Math. Sci., 25 (1989), 681-740.

[18] M. Tomari AND K.-I. Watanabe, Normal $Z_{r}$-graded rings and normal cyclic covers, Manuscripta Math., 76 (1992), 325-340.

[19] J. M. WAHL, Equations defining rational singularities, Ann. Sci. École Norm. Sup., 10 (1977), 231-264.

[20] K.-I. Watanabe, Some remarks concerning Demazure's construction of normal graded rings, Nagoya Math. J., 83 (1981), 203-211.

[21] K.-I. Watanabe, Rational singularities with $k^{*}$-action, Commutative Algebra (S. Greco and G. Valla eds.), Lecture Notes in Pure and Appl. Math. 84, Dekker, New York, 1983, 339351.

[22] K.-I. Watanabe, Regular Z-graded rings, Proc. Fac. Sci. Tokai Univ., 25 (1990), 1-8.

Department of Mathematics

FACULTY OF SCIENCES

KANAZAWA UNIVERSITY

KaKuma, KanaZaWA, 920-1192, JAPAN

e-mail: tomari@kenroku.kanazawa-u.ac.jp

Department of Mathematics

College of Humanities and Sciences

NiHON UNIVERSITY

Setagaya, Tokyo, 156-0045, JAPAN

e-mail: watanabe@math.chs.nihon-u.ac.jp 